\title{
THE ELECTIVE LOCALIZATION OF STREPTOCOCCI FROM EPIDEMIC POLIOMYELITIS
}

\author{
Plates 9-13
}

E. C. Rosenow, E. B. Towne, ANd C. L. v. Hess

From the Mayo Foundation, Rochester, Minn.

It is our purpose in this paper to review the bacteriology of poliomyelitis, to record experiments on the elective localizing power of bacteria isolated from the supposed infection-atrium and infected tissues in cases of poliomyelitis which occurred during the epidemic of 1916, and to discuss briefly the significance of the findings.

Many attempts have been made to produce lesions in the central nervous system, especially myelitis, by the intravenous injection of bacteria. Roger injected streptococci in rabbits. Emaciation and muscular atrophy resulted, and there was degeneration of the ganglion cells in the spinal cord, but bacteria were not demonstrated. Bourges, working with the streptococcus from erysipelas, discovered that one of his rabbits developed paraplegia, paralysis of the sphincters, and muscular atrophy. At necropsy, 15 days after inoculation, no lesions were found except in the spinal cord which showed hyperemia, hemorrhages, neurogliosis and nerve-cell degeneration, but no streptococci. Morel and Rispal had one case of experimental myelitis in which, 7 days after inoculation, streptococci were found both in the blood and in the spinal cord. In a large series of animals (116), Vidal and Besanzon observed paralytic symptoms in seven. Only degenerative lesions of the spinal cord were found. No streptococci were discovered in sections or cultures. Poynton and Paine recovered streptococci from the cerebral fluid in one rabbit after intravenous injection. No pathology or symptoms were mentioned. Wickman ${ }^{51}$ attempted to produce a special affinity of streptococci for the central nervous system in rabbits by repeated passage through the central nertous system, but failed. Similar results were obtained by Marinesco. Hoche and others could obtain lesions of the spinal cord on intravenous injection of bacteria only by the simultaneous injection of inert powders.

Davis, Horder, Henrici, Moody, Irons, Brown and Nadler, Gerdine and Helmholz, Hardt, Detweiler and Robinson, Blake and others, who have injected numerous animals intravenously with streptococci from sources other than the nervous system, have not reported the occurrence of flaccid paralysis due to lesions in the central nervous system. Bull produced meningitis in dogs by injecting intravenously large doses of virulent pneumococci. In other animals paraly. sis, apparently of the spastic type, developed, but the occurrence of flaccid paralysis is not mentioned.

During elective localization studies made by one of us, ${ }^{44,45,46}$ some 2,000 rabbits and guinea-pigs were used, excluding those injected with bacteria isolated from diseases of the nervous system. Sixty per cent 
of these animals lived from 1-3 days, $20 \%$ from $3-7$ days, $10 \%$ from 7-20 days, and 10\% 20 days or longer. Only three showed definite paralysis, which was proved not to be a general weakness due to overwhelming infection.

Weakness, especially of the hind extremities, shortly before death was noted occasionally, but this was taken to be a part of an overwhelming infection.

It is evident, therefore, that bacteria obtained from miscellaneous sources rarely localized in the central nervous system following intravenous injection. Even in some of the experiments considered positive, paralysis alone was the basis of diagnosis with no necropsy to rule out other causes. Frequently the microscopic examination was unsatisfactory and bacteria usually could not be found. The concensus of opinion has been ably expressed by Buzzard: "The whole of these researches illustrate the difficulty which is encountered in producing experimentally an infective condition of the central nervous system through the general circulation, an experience which is not without parallel in clinical medicine, when we consider how comparatively rarely ordinary pyogenic organisms are the cause of disease within the brain and particularly within the cord unless as a direct infection from the neighboring tissue."

The isolation of bacteria in acute poliomyelitis has been reviewed by various authorities (Wickman, ${ }^{s 1}$ Landsteiner, Römer ${ }^{11}$ and others). Schultze, in 1898, found diplococci in the spinal fluid by spinal puncture in a case 13 days after the onset. Cultivation on glycerin agar was negative. Following this, Bülow-Hanson and Harbitz, Concetti, Looft and Dethloff, and Engel, each of whom reported one or two cases, found a similar organism.

Giersvold, who studied the epidemic in Norway in 1905-1906, isolated a grampositive polymorphic diplococcus from the spinal fluid in each of 12 cases of poliomyelitis, in some instances both before and after death. He noted that the later in the disease the spinal puncture was performed the fewer were the bacteria. After from 12-14 days the spinal fluid was often sterile. He stated further, that the isolated microorganisms, except in one case in which there were also pneumococci, were obtained in pure culture, that they were bean-shaped diplococci or tetracocci, often in clumps when grown on solid mediums, and in chains of 4-6 diplococci when grown in liquid mediums, the line of division between the pairs corresponding with the axis of the chain, and that the growth in the first culture generation was usually slight but became richer on transplantation. Paralytic symptoms were noted following injection in mice and rabbits in a few instances.

Fox, ${ }^{12}$ in 1907 , found gram-positive and biscuit-shaped diplococci and tetracocci in the cultures from the nose and throat of all his acute poliomyelitis cases. The spinal fluid which was obtained in four instances showed the same organism. In another epidemic in $1910,{ }^{13}$ the gram-positive diplococcus was found in smears from the inner portions of the brain and cord of 3 cases in man, where, it is 
said, "there could be no possibility of contamination from the outside." Pasteur, Foulerton and MacCormac found the same organism in the spinal fluid in one case. No intravenous inoculation into animals was made.

Potpeschnigg, in 14 cases, found the same diplococcus both in smears and cultures of the spinal fluid although later he decided they were contaminations. In 1910, "Giersvold's diplococcus" was found by Robertson and Chesley in the spinal fluid in 7 cases and also in the brain in one fatal case. Three-tenths of a c c of a growth from agar-slants injected intravenously into each of several animals, gave paralytic symptoms in two. Poliomyelitic lesions were not found in the spinal cords. The same diplococcus was recovered from the cerebrospinal fluid of some of their animals.

About this time Landsteiner and Popper, Flexner and Lewis, Römer, ${ }^{42}$ and Leiner and Wiesner, each succeeded in producing typical poliomyelitis in monkeys with virus, and later with filtrates of virus. Flexner and Noguchi, in 1913, cultivated minute "globoid" bodies from the brain and cord which, when injected intracerebrally in monkeys, produced typical lesions of poliomyelitis, and which they state "fulfill the conditions hitherto demanded for the establishment of causal relationship between an extraneous parasite and a specific disease." They considered cultures contaminated when containing bacteria of ordinary size. To quote: "Any tube showing marked turbidity, coagulation, or gas-production within one or two days may be set aside as grossly contaminated. The tubes which remain clear or show only slight turbidity may none the less be contaminated. To exclude contamination, small quantities of the medium are removed with pipettes and stained for bacteria in the ordinary way and cultivated on the usual solid or fluid media. Obvious bacteria in the films as well as any growth taking place in the ordinary media in the presence of air, may be put down as contaminations and the tubes containing them discarded."

From that time, bacteria of ordinary size have been considered of little or no importance in the etiology of acute poliomyelitis.

In attempting to determine causal relationship of the bacteria isolated from poliomyelitis, investigators have usually injected relatively small doses of culture from agar slants or from low columns of bouillon. Little or no attention was paid to the question of oxygentension, temperature of incubation, and the length of time organisms were grown on mediums before injection. The importance of these factors has been emphasized in articles by Rosenow ${ }^{44,45,46}$ in connection with a study on the etiology of various diseases, including diseases of the nervous system. By affording bacteria a gradient of oxygenpressure at a relatively low temperature (33-35 C.), and by injecting a large number of organisms soon after isolation, there was observed 
a marked tendency for the bacteria to localize and produce lesions in the tissues of animals corresponding to those involved in the spontaneous disease.

On the basis of these experiments the completely negative or largely negative results of the earlier investigations were considered inconclusive as proving that the diplococci or streptococci found by many observers in poliomyelitis, were contaminations and therefore of no etiologic importance. Accordingly it was decided to reinvestigate the bacteriology of epidemic poliomyelitis and to determine any specific localizing power of the bacteria isolated by means of the newer methods.

From July 21 to Aug. 12, 1916, the localizing power of cultures from tonsils in 7 cases of poliomyelitis that occurred in Rochester, Minn., were studied. Paralysis and corresponding spinal lesions were produced in some animals with cultures from all 7 cases (Figs. 1, 2, 10 and 14). A pleomorphic streptococcus was found to be the organism which showed specific localizing power. On the basis of these results the work was continued in New York where postmortem material might be studied.* The experiments with tonsil-strains were repeated with similar results and Aug. 21 the first series of animals was injected with the pleomorphic streptococcus isolated in pure culture from the spinal cord in a typical case of poliomyelitis (Pig 324). A description of the pleomorphic streptococcus isolated from the infection-atrium in numerous cases and from the central nervous system or lymph glands in each of 12 cases and its elective affinity for the nervous system was given in a preliminary report.

Mathers, ${ }^{30}$ and Nuzum and Herzog have reported similar results using methods which embody essentially the points which had been emphasized repeatedly by Rosenow in elective localization studies, even to the point of incubating cultures for injection at a relatively low temperature $(35 \mathrm{C})+.\dagger$ Mathers ${ }^{31}$ reported definite paralysis in 10 of 18 young rabbits injected with cultures from 1 case, and gross and microscopic changes similar to those in acute poliomyelitis in man in 15 . Hektoen has shown that the microscopic changes are indeed quite like those in man. Nuzum and Herzog inoculated 50 rabbits and reported the development in a considerable number of "a most striking type of progressive flaccid paralysis involving both hind legs, the front legs,

* Note-Facilities for this study were kindly furnished by the New York Hospital.

† Since the question of priority has been raised (Current Comment, Jour. Am. Med. Assn., 1916, 67, p. 1235), it may be stated that the experiments reported in this paper were begun 6 weeks before those of Mathers with strains from tonsils, and 2 weeks before
his with strains from brain and spinal cord, and 7 weeks before those of Nuzum and Herzog, according to published reports. 
only one leg, or, in 3 cases, all four legs." Histologically, the changes observed by them "differ from the human in that no perivascular infiltration is present and that the types of infiltrating cells are probably not lymphocytes." Flaccid paralysis was also produced in young dogs but repeated injections in guinea-pigs resulted negatively in their hands. That the positive results obtained by Rosenow, Towne and Wheeler, ${ }^{48}$ by Mathers, and by Nuzum and Herzog are due to the methods employed is supported by the fact that Bull, and Kolmer, Brown and Freese $^{22}$ have recently reported negative results obtained by methods similar to those used by the earlier investigators and which do not take into account sufficiently the points essential for elective localization. Thus, the results obtained by Kolmer, Brown and Freese may be accounted for by the aerobic cultivation of the bacteria, the smallness of the dose and the use of adult rabbits (1,400-1,600 gm.).

\section{TECHNIC}

The technic was essentially that used in elective localization studies in other diseases. Painstaking effort was made to express the material containing the bacteria from the depths of the tonsils. This was done by means of a small laryngeal mirror straightened to an angle of about 30 degrees with the holder. The material was scooped out and then swabbed from the mirror with a sterile cotton swab. Surface bacteria were eliminated as far as possible by having the patient drink water or gargle with some antiseptic solution just before examination. Extirpated tonsils were thoroughly washed in sterile salt solution and then cut into thin slices and emulsified in salt solution. The infected material from abscesses was suspended in salt solution and then inoculated into the various mediums, and in some instances directly injected into animals. The infected tissues, brain, spinal cord and lymph glands, were obtained in a sterile condition, emulsified usually in a sterile air chamber and then inoculated into mediums and, in some instances, injected intracerebrally into animals.

The bacteria for injection were usually grown under partial oxygentension in tall columns $(9-10 \mathrm{~cm}$.) in bottles, approximately $150 \mathrm{c} \mathrm{c}$ capacity, of ascites-dextrose, or of dextrose broth $(0.2 \%$ dextrose; $0.6 \%$ acid to phenolphthalein) with or without tissue. The cultures were incubated at from 33-35 C. for from 18-24 hours and occasionally longer. The primary culture was chiefly used, although the second, third, and fourth culture generation from single colonies were injected in some instances. Injections were made of the broth culture or the 
bacteria suspended in salt solution. In some instances cultures in ascites-tissue fluid were injected. The dose for the broth culture was usually from $0.5-3 \mathrm{c}$ c for guinea-pigs and $2-5 \mathrm{c}$ c for rabbits. The suspensions for injection were made so that $1 \mathrm{c} c$ of the salt solution contained the growth from $15 \mathrm{c} \mathrm{c}$ of the broth culture, the dose of the suspension being $0.5-3 \mathrm{c} \mathrm{c}$ for guinea-pigs and $2-5 \mathrm{c} \mathrm{c}$ for rabbits. The injections in rabbits were made into the marginal ear-vein, those in the guinea-pig, dog, and cat into a leg-vein, the inoculations being made quite rapidly through a 21 or 23 gatige needle. A blood-agar plate culture was made of all suspensions immediately after injection to prove viability and kind of organism. Only animals from perfectly healthy stock were used. Young animals in each species were chiefly selected. The animals were examined two or more times daily for evidences of arthritis, myositis and other lesions, and for symptoms referable to the central nervous system. Chloroform was used to kill the animals for examination. Necropsies were made as soon as possible after death. Careful search was made for lesions at the point of injection, and of joints, muscles and nerves to make sure that the symptoms observed during life were not due to these causes. Cultures were made on blood-agar plates and in tall columns of dextrose broth $(15 \mathrm{cc})$ as a routine from blood $(0.1-0.5 \mathrm{cc})$ and the aspirated brainsubstance containing more or less ventricular fluid $(0.1-0.3 \mathrm{c} \mathrm{c})$, and in numerous instances from other tissues.

The brain and cord were removed and placed into $10 \%$ formalin or into Kaiserling solution for $12-48$ hours. Numerous cross sections at levels of about $3 \mathrm{~mm}$. were then made in search of lesions. The portions put aside for sections for microscopic study consisted as far as possible of blocks showing gross lesions. The above solutions and Zenker's solution were used as fixing fluids. The tissues were embedded in paraffin and the sections stained with hematoxylin and eosin, with methylene blue and eosin and by the Gram-Weigert and Giemsa methods for bacteria.

\section{Illustrative Protocols of Animals After Intravenous Inoculation}

Group 1.-Guinea-pigs.-Guinea-pig 262, weight 200 gm., was injected Aug. 2. 1916, with $2 \mathrm{c}$ c of ascites-dextrose broth culture of the tonsil from Case 695.

Aug. 3, the animal seemed well but there was undoubted weakness of the extensors of the right hind leg. The next day it was reinjected with washings from a scant growth on two blood-agar plates. Aug. 5, it was weak in the hind extremities and in the muscles of the back (Fig. 1). This was more pronounced the next day, but by Aug. 8 it had regained almost normal strength in the extremities and muscles of the back. The animal was chloroformed. 
Cultures of the blood were sterile. No gross lesions were found and no cultures were made from the brain and cord. Microscopically, there were found thrombosis of the veins of the surface and interior of the lumbar cord, localized areas of hemorrhage in the cord, moderate degeneration of the ganglion cells and neurophagocytosis in areas. Diplococci were present in the areas of hemorrhage in the cord and those surrounding the spinal ganglia.

Guinea-pig 264, weight $250 \mathrm{gm}$., was injected Aug. 3, 1916, with $2 \mathrm{c}$ c of ascites-dextrose broth culture (18 hours) of streptococci obtained from a single colony of blood-agar plate from the tonsil pus of Case 695. The next morning the animal appeared well. At $1 \mathrm{p} . \mathrm{m}$. it had marked tremor and weakness of the left front leg. At 4:30 p. m. the weakness in the left front leg was increased, there was general tremor over the whole body; the animal was very excitable. and was moving about constantly. When the hind legs were held the tremor was constant. moderately coarse, and very marked. The left foreleg dragged with a complete toe-drop. The thorax was kept from the floor with difficulty, due to the weakness and there was rapid fatigue of the extensors of the left foreleg. The right foreleg was fairly normal. Following moderate exercise toe-drop developed; with more activity the entire leg dragged. While a moving picture was taken a similar condition gradually developed in the right hind leg so that finally the animal lay with both hind legs stretched out behind. With exhaustion the tremor became more marked, and twice there was a true convulsion of the left hind leg lasting from 30-40 seconds. Reflexes were not obtained. The tone of the muscles of the left foreleg was decreased. At 5:30 p. m. there was complete extensor paralysis of both the hind legs and the left foreleg. There was a repeated twitching of the left posterior hamstring muscles and sharp contractions of the diaphragm, resembling a hiccough. At 5:45 p. m. a general spastic condition with a tendency to retraction of the head developed and there were a number of general convulsions. Dyspnea was marked and the breathing was entirely diaphragmatic. Death occurred from respiratory failure at $6 \mathrm{p} . \mathrm{m}$.

Examination showed a marked hyperemia of the extradural plexus, with softening and edema of the cord, and hemorrhages of the anterior surface of the medulla and pons. There were hemorrhages of the anterior root of the left third dorsal nerve with small hemorrhages in the left sciatic nerve, and edema of the legs. Cultures in blood-agar plates of the cervical cord, brain, lumbar cord, and muscles showed a moderate number of very fine, dry, slightly greenproducing colonies of streptococci. Cultures from the blood, spinal fluid, kidney and liver were sterile. Numerous cross sections of the brain and cord, hardened in formalin, showed marked hyperemia of the vessels of the gray matter of the cord, together with a number of small hemorrhages, but no lesions in the brain. Microscopically, a mild meningitis and degenerative hemorrhagic lesions in the brain and spinal cord, and streptococci in the lesions were found.

Guinea-pig 266, weight $240 \mathrm{gm}$., was injected Aug. 4, 1916, at 9 a. m. with $2 \mathrm{c} \mathrm{c}$ of ascites plain broth culture (18 hours) from the tonsil of Case 695 . The same day at 9:30 p. m. the animal appeared sick, uncomfortable, and irritable. The extensors of the hind legs were weak and walking was difficult. The hind legs slipped back and constant effort was made by the animal to get them under it. The muscle-tone of the hind legs was diminished. No resistance was felt in the extensors of the wrists and knees. Hiccough occurred at intervals. Aug. 5, the animal was found dead.

A number of hemorrhages had occurred in the stomach, cecum, papillary muscles of the left ventricle, and the liver, and a few in the lungs. There were edema and congestion of the pia. and hemorrhage and edema surrounding the dura and spinal ganglia. The lymph glands were hemorrhagic. There was 
edema in the region of the solar plexus. No lesions were found in the nervetrunks, joints or muscles. Cultures from the brain-substance showed greenproducing streptococci, those from the blood showed a few colon bacilli but no streptococci. Microscopically, there were found a mild meningomyelitis with degeneration of ganglion cells and a moderate number of gram-positive diplococci in the hemorrhagic areas surrounding the spinal ganglia.

Guinea-pig 270, weight $260 \mathrm{gm}$., was injected Aug. 4, 1916, at 9 a. m., with the growth from $30 \mathrm{c} \mathrm{c}$ of ascites plain dextrose broth culture from the tonsil of Case 695. At 9:30 p. m. the symptoms were almost exactly like those of Guinea-pig 266. The animal appeared sick, uncomfortable and was very irritable. There was weakness of the extensors of the thighs and legs. The hind legs could not be kept from slipping back, and it constantly made efforts to get them under the body. In walking it could not come up on its toes. There was occasional twitching of the muscles of the back and the hind legs and the latter tended to spread apart as though the adductors were weak. Walking required great effort. There was occasional hiccough. Aug. 5, the animal was found dead.

There were hemorrhages in the papillary muscles of the left ventricle, in the liver, stomach, cecum, and a few in the lungs. The mesenteric glands were hemorrhagic and there was marked edema in the retroperitoneal space and about the pancreas. The cord was very soft. There was marked congestion of the vessels of the pia, and hemorrhage and edema surrounding the cord and ganglia. Cultures showed from the brain a large number of streptococci, varying greatly in size, shape and grouping, and from the blood a smaller number together with colon bacilli. The microscopic sections showed focal hemorrhages and early infiltrative meningitis and myelitis, chiefly with round cells. Streptococci were demonstrated in the meninges and in the hemorrhages in the gray matter of the cord.

Guinea-pig 324, weight 100 gm., was injected Aug. 21, 1916, at 9 a. m., with $0.5 \mathrm{c} \mathrm{c}$ of ascites-dextrose broth culture inoculated with a single colony in ascitesdextrose-agar, originally isolated from the spinal cord of Case 707 . At $5 \mathrm{p} . \mathrm{m}$. there was marked tremor of the legs, and marked opisthotonos. The animal seldom moved the left hind leg, rarely extended the left hind leg or foreleg, and then weakly. It could not stand. The muscle-tone of the left hind leg was much below normal. At $9 \mathrm{p}$. $\mathrm{m}$. the animal was found lying on its side with head retracted, and having convulsive spasms at intervals of from a few minutes to 10 minutes. There were coarse tremor and clonic spasms in the muscles of the extremities and of the eyelids. It was unable to stand and pawed the air with the legs, always less actively with the left hind leg. At 9:30 p. m. the condition was much the same. The temperature was $97.6 \mathrm{~F}$. There were gen. eralized convulsions with the head markedly retracted and marked twitching of the muscles of the face. At 10 p. m. it was etherized.

The brain and cord were quite soft. The pia appeared opaque. The cerebrospinal fluid was clear. There was a moderate amount of clear fluid in the peritoneal cavity, and the mesenteric lymph glands were edematous and hemorrhagic. There was marked edema of the retroperitoneum in the region of the splanchnic area and about the pancreas. No lesions were found in the viscera. Cultures from the blood showed a few, while the spinal fluid and the brain showed countless numbers of very fine, slightly green-producing colonies of streptococci. Cultures from the kidney were sterile. Microscopic sections showed chiefly meningitis with round cells predominating. There was marked congestion of the pia and the vessels of the gray matter, with slight perivascular infiltration, degeneration of the nerve cells and areas of edema and hemorrhage in the gray matter, marked degeneration of the nerve cells, and neurophagocytosis. 
Guinea-pig 337, weight 195 gm., was injected Aug. 23, 1916, with the growth from $3 \mathrm{c} c$ of ascites-dextrose broth culture of streptococcus from the spinal cord of Guinea-pig 324. Aug. 25, the animal appeared well but showed weakness in the right hind leg. It was reinjected with ten times the original dose. Aug. 26, it appeared quite well but was weak in both hind legs, the weakness being more marked in the left. Aug. 27, the weakness had extended to the left front leg. The temperature was $104.6 \mathrm{~F}$. Aug. 31 , the weakness in the hind legs was still present. The animal was just able to get the hind legs under it but was scarcely able to walk. Both hind feet tended to turn outward, while the weakness in the left front leg was less marked. Sept. 2, the animal had grown very thin but was taking more food and its general condition was better. Weakness was most definite in the extensors of the left hind leg, and perhaps also in the adductors. There was no arthritis and a few steps could be taken slowly. Sept. 6 , the condition was about the same. The left hind leg was very weak. The hind quarters frequently dropped to the left. There were contractures of the muscles and inability to extend completely the left knee and ankle-joints (Fig. 3). Sept. 7, at 4 p. m. the animal was found dead.

There was marked atrophy of the muscles of the hind legs out of proportion to the general wasting. The contents of the large and small bowel were fluid. There was an area of marked edema and hemorrhage in the sigmoid $12 \mathrm{~cm}$. from the rectum, due to partial intussusception. There was edema in the retroperitoneum about the pancreas, and a few small hemorrhages in the cortex of the kidney. There were no lesions of the joints. The vessels of the pia were hyperemic, and the brain and cord were soft. Cultures from the brain, cervical cord and lumbar cord showed fine, dry, green-producing colonies of streptococci, those from the blood and liver remained sterile, and those from the kidney showed many green colonies of streptococci.

Guinea-pig 352, weight $165 \mathrm{gm}$., was injected Aug. 24, 1916, with 2 c c of ascites-fluid culture in the second generation from the spinal fluid of Guinea-pig 324. Aug. 25, the animal appeared quite well, but was spastic and weak in both rear and the left front extremities. It was reinjected with growth from $30 \mathrm{c} \mathrm{c}$ of ascites plain broth culture. Aug. 26, it appeared better, though weakness in the left hind leg was still noticeable. Aug. 28, it was found dead.

There were a few lesions in the muscles, a mild embolic nephritis, and a number of sharply circumscribed hemorrhages beneath the dura of the cord and the anterior surface of the medulla. Cultures from the brain showed an enormous number of fine dry, green colonies of streptococci, while those from the blood showed ten moist green colonies. Numerous cross sections of the brain and cord showed a number of localized hemorrhages in the brain. Microscopically, there were found a diffuse mixed-cell meningitis, hemorrhages, degeneration and slight infiltrative changes in the brain and cord, and a latge number of streptococci in and adjacent to the areas of hemorrhage (Fig 8).

Guinea-pig 374, weight $190 \mathrm{gm}$., was injected Aug. 26, 1916, at 5:30 p. m., with the growth from $22.5 \mathrm{c} \mathrm{c}$ of ascites-dextrose broth culture of streptococcus from the brain substance of a cat injected intracerebrally with emulsion of the brain of Case 714. At $10 \mathrm{p}$. $\mathrm{m}$. the animal seemed weak in the left hind leg. Aug. 27, at 9 a. m., it was weak in both hind legs, especially the right, carried its weight on the left side and often dropped quickly to the right. No difference in the strength in the forelegs could be made out. At 10:30 a. m. it was tremulous, the weakness in the hind legs was more marked and had extended to the right front leg; it was just able to walk. At 7 p. m. the hair was ruffled, the respirations were increased, the temperature was $101.8 \mathrm{~F}$. and weakness in both front legs was evident. Aug. 28, it was found dead. 
There were hemorrhages and edema surrounding the cord, and marked hyperemia of the vessels of the meninges and gray matter of the cord. Cultures from the brain substance in ascites-dextrose broth showed pure growth of the pleomorphic streptococcus. Microscopic sections showed marked hemorrhagic and infiltrative leptomeningitis, and marked disseminated infiltrative encephalomyelitis, with perivascular infiltration, advanced nerve-cell changes, round-cell invasion, and a large number of streptococci in the infiltrated and hemorrhagic areas (Figs. 6 and 7 ).

Guinea-pig 384, weight 220 gm., was injected Aug. 27, 1916, with the growth from $10 \mathrm{c} c$ of ascites-dextrose broth from streptococci from the brain substance of a guinea-pig injected intravenously with a culture from the tonsil in the second culture from Case 712. The animal seemed quite well until Aug. 30, when it was weak in the left foreleg, and both hind legs, was just able to walk and tended to fall to the left side. There was no swelling of the joints or at the point of injection. Oats were eaten and water drunk. It was unable to draw the hind legs under it (Fig 4). Sept. 2, the animal was very thin but ate well and the general condition was good. It walked a little and could pull its hind legs under it. There was marked weakness of the muscles of the back. When it was laid on one hip or the other with the front legs under the body, it could not raise its hind quarters. Abductor weakness was apparent in the left thigh, and more marked extensor weakness in the right hind leg. The extensors of the front legs were quite normal. Sept. 7, it ate well, appeared better generally, could get up when lying on either side though weakness was still most marked in the extensors of the right hind leg. Sept. 19, it was found dead; weight, $150 \mathrm{gm}$.

There was marked atrophy of the muscles of the hind extremities, especially the quadriceps, and marked contracture of the hamstrings on both sides. There were no gross lesions. Cultures were negative. Microscopic sections showed degenerative and infiltrative changes in the meninges and cord. A few diplococci were found in the meninges.

Guinea-pig 396, weight $170 \mathrm{gm}$., was injected Aug. 29, 1916, at 5:45 p. m.. with growth from $15 \mathrm{c} c$ of ascites plain broth of the streptococcus from the brain of Guinea-pig 374. Aug. 30, at 8 a. m., the animal was excitable, tremulous. and definitely weak in the left hind leg. At $5 \mathrm{p}$. m. it fell constantly to the left; both hind legs were weak, especially the extensors, and it was barely able to advance its legs forward under the body. It seemed in pain when handled. There was undoubted weakness in the left foreleg. Aug. 31, at $12 \mathrm{~m}$. both hind legs were weak, especially the right, which it could not draw forward (Fig. 5). Sept. 2, the animal seemed much better, and got about well on three legs. The left hind leg was held up, suggesting arthritis, though this condition could not be made out. It was still irritable. There was marked extensor weakness of the left hind leg, but it could pull its legs under it. The right hind leg seemed normal. Sept. 5, it was found dead.

There was marked myocarditis, acute splenitis, arthritis of the left ankle, and marked softening of the brain and spinal cord. Cultures from the brain, spinal cord and blood showed streptococci. There was marked meningitis with extension into the adjacent nerve-tissue. There were hemorrhages and degeneration of the brain and cord, and many diplococci in and adjacent to the lesions. The infiltrating cells were chiefly mononuclear.

Guinea-pig 422, weight $200 \mathrm{gm}$., was injected Sept. 2, 1916, with the growth from $15 \mathrm{c} \mathrm{c}$ of ascites-dextrose broth inoculated with the emulsion of the tonsil of Case 722. The next day the animal was very excitable, shaky and tremulous; it rushed blindly about the cage, and fell frequently, always to the left. The head was retracted. Both left legs were weak. There was purulent conjunctivitis in both eyes. Sept. 4, it was found dead. 
Myocarditis, slight edema of the lungs, marked hemorrhage and edema surrounding the dura of the lower dorsal and lumbar cord and hyperemia of the vessels of the pia were found. Cultures on blood-agar plates from the blood were sterile, but from the lumbar cord and brain they showed a few fine green colonies of streptococci. Microsscopically, there was found a predominating meningitis with mononuclear and polynuclear cells and slight infiltrative but marked degenerative lesions in the brain and cord. Streptococci were easily demonstrated in the areas showing lesions.

Guinea-pig 430, weight 180 gm., was injected Sept 5, 1916, with the growth from $30 \mathrm{c} \mathrm{c}$ of ascites-dextrose broth culture of small green-producing streptococcus from the tonsil in Case 722 in the third culture. The next day the animal apparently had a peculiar tic; the head was turned to the left and jerked constantly about 180 times per minute, the jaws working at the same rate. It was very irritable and excited, and was constantly on the move, turning to the left. Hay was taken in the mouth, but not swallowed. The front legs were fairly steady but the hind legs were weak and perhaps ataxic. The hind quarters frequently fell to the right, but an upright position could nearly always be regained. There was definite extensor weakness of the hind legs, especially the right; probably also abductor weakness. There was no spasticity and no nystagmus. Sept. 8, the weakness of the left hind extremity was more marked, the extensors were about the same, the animal walked weakly, and the hind quarters tended to drop to the left. There was prolapse of the rectum which was easily reduced. Oats were eaten. It was found dead the following day.

Necropsy showed hemorrhage and edema about the prolapsed portion of the rectum. There was hyperemia of the vessels of the pia and hemorrhage and edema surrounding the dura and cord; no lesions of the viscera. Cultures from the kidney, brain, cord, blood ar d liver showed colon bacilli, evidently due to infection from the rectum. Berkefeld $\mathrm{N}$ filtrates of the brain emulsion yielded pure culture of the pleomorphic streptococcus. Microscopically, lesions of the centrál nervous system were similar to those found in Guinea-pig 422.

Group 2.-Rabbits, Dogs and Cats.-Rabbit 962, weight $670 \mathrm{gm}$., was injected Aug. 20, 1916, with the growth from $30 \mathrm{c}$ c of ascites-dextrose broth culture from the secretions in the throat and tonsil of Case 706. The following day the animal seemed quite well but was definitely weak in the hind extremities. Aug. 25, at noon, it was found lying on the right side with the head and eyes turned far to the right, and there was marked nystagmus. The right fore and hind legs were very weak, the left being less so and spastic. It was unable to stand and when placed on the left side it quickly flopped to the right, but when placed on the right side it could not get up. The muscle tone and reflexes on the right side were diminished. The left front leg was almost spastic. In the afternoon it was very weak, just able to walk. It drank water and ate oats. The following day the weakness was less marked and the temperature was 104 F. Aug. 28, it was found dead.

Vegetative, aortic and mural endocarditis, a few embolic abscesses and infarcts in the kidneys, hemorrhagic ederna surrounding the dura of the cord. subdural hemorrhages between the cerebellum and cerebrum, and on the anterior surface of the medulla, were found. The fluid in the anterior chamber of the right eye was cloudy. Cultures from the blood and kidney showed a few colonies of staphylococci. Those from the brain gave a pure growth of rather moist green colonies of streptococci. The microscopic sections showed slight infiltration of the meninges and the gray matter of the spinal cord, marked degeneration of nerve cells, especially in the anterior horns, and streptococci in the pia. 
Rabbit 977, weight $420 \mathrm{gm}$., was injected Aug. 24, 1916, with $6.5 \mathrm{c}$ c of the second culture-generation of the spinal fluid of Guinea-pig 324. The following day the legs were weak, especially on the left side. If lying on the right side it could get up, but if on the left side it could not do so. It remained quiet unless started, when it would go a little way fairly normally and then become exhausted. Respirations were normal and it did not appear sick otherwise. It was chloroformed.

A moderate number of subperitoneal hemorrhages in the appendix and sigmoid, and a small amount of fluid blood in the peritoneal cavity were found. There were hemorrhages in the gallbladder and a large number of circumscribed submucous hemorrhages in the pyloric end of the stomach. There was marked edema surrounding the dura of the spinal cord, cspecially the cervical and lumbar enlargements. The cerebrospinal fluid was increased in amount and slightly blood-tinged. The vessels of the pia were markedly congested. There were a few small hemorrhages in the right sciatic nerve, in the left external popliteal space, and in both posterior tibial nerves; a few small hemorrhages and edema in the muscles about both hip joints, marked hemorrhage in one of the left gluteal nerves, and a few small hemorrhages in the nerve trunks of the forelegs. Cultures from the blood, brain and peritoneal fluid showed pure cultures of the green-producing streptococcus.

Rabbits 980 and 981, weights $410 \mathrm{gm}$. and $350 \mathrm{gm}$., were injected Aug. 25, 1916, with 3 c c, respectively, of broth culture from the tonsi: emulsion from Case 714 . Eight days later Rabbit 980 developed a peculiar gait caused by marked weakness of the adductors of the left hind leg. Eleven days after injection the extensors of both hind legs were weak. Gradual recovery took place during the following week. It died on the 20 th day. Rabbit 981 remained apparently well until the 19th day when it was found lying on its side, moving the head very little and the extremities not at all. Respirations were slow, laborious and were almost wholly diaphragmatic. The animal died, apparently of respiratory failure, on the same day.

In both animals there was marked increase of cerebrospinal fluid; hemorrhage and edema surrounding the dura, congestion of the vessels of the pia, but no lesions of viscera, joints, or muscles and nerve trunks. Cultures of Rabbit 980 from the brain, lumbar cord, blood, liver and kidney were negative. Of Rabbit 981, cultures on blood-agar plates of the spinal fluid, brain, lumbar cord, liver, and blood were negative, but cultures in ascites-dextrose broth of the brain and blood yielded pure culture of a markedly pleomorphic streptococcus. Microscopic sections of both showed a slightly infiltrative meningomyelitis. At the medullary, cervical, and lumbar levels the meninges showed hyperemia, hemorrhages, a few round cells, and an occasional diplococcus. Lesicns in the gray matter were more evident and consisted of hyperemia, numerous focal hemorrhages, slight adventitial and diffuse infiltration with round cells, increase of the neuroglial cells, nerve cell degeneration, satellitosis, neurophagocytosis and streptococci and small round cells (Fig. 18). The white matter showed slight gliosis and similar lesions were found in the spinal ganglia. The brain showed no infiltration.

Rabbit 982, weight $260 \mathrm{gm}$., was injected Aug. 25, 1916, with the growth from $22.5 \mathrm{c} \mathrm{c}$ of the culture from the tonsil emulsion of Case 714. Aug. 27, there was lacrimation and circumcorneal injection. The fluid in the anterior chamber of the eye was cloudy. Aug. 28, 8 a. m., there was a coarse tremor of the whole body, marked weakness of the front legs, but the animal was able to walk. At noon the tremor was much the same, the weakness in the frontlegs, especially the right, had increased. At 5:30 p. m. the right front leg was flaccid; the left was very weak, but could be used a little. It could not lift the thorax, head or 
hips off the floor of the cage. Respirations were entirely diaphragmatic. There was considerable weakness but no paralysis of the hind legs. The tremor had disappeared. At $10 \mathrm{p}$. m. it was found dead.

The pericardial sac contained a small amount of turbid fluid and fibrin flakes. There were a few white muscle lesions in the inner aspects of the right thigh and about the right hip. The fluid in the anterior chamber of both eyes was turbid. There were beginning tricuspid endocarditis, marked edema of the dura and surrounding tissues, hemorrhage surrounding the spinal nerve roots, marked hyperemia of the vessels of the pia and hemorrhages in the gray matter of the cord. Cultures from the brain, blood and anterior chamber of the eye showed pure growths of greenproducing streptococci resembling pneumococci, while cultures from the pericardial fluid showed pure growth of hemolyzing streptococci. Microscopic sections showed a moderate, chiefly mononuclear, leptomeningitis more marked over the brain, and degeneration and gliosis in the brain and cord, with slight infiltration of the gasserian ganglion. Small hemorrhages were seen in the brain and medulla only. The choroid plexus showed vascular dilatation, edema and hemorrhage. Diplococci were found in the lesions.

Rabbit 985, weight $630 \mathrm{gm}$., was injected Aug. 26, 1916, with $4 \mathrm{c} \mathrm{c}$ of culture of the brain of Rabbit 979 which was injected intracerebrally with the emulsion of tonsil of Case 714. The second day at $4 \mathrm{p}$. m. the animal was very excitable. When put on the floor it rushed about toward the right in three-foot circles. After a minute or so the right front and hind legs gave out, it fell and was unable to get up again. Respirations were rapid. After resting it was evident that use of the abductors and muscles of the right hind leg was completely gone so that the leg remained crossed under it. The extensor muscles of the right hind leg were so weak they could not be brought forward. The left hind leg also showed abductor weakness and the leg crossed under it, but to a less extent than the corresponding right. The right foreleg was weak, and after exercise lay out motionless perpendicular to the body. The left foreleg was also weak but could be used. The head tended to fall to the right. Two convulsions occurred after which the respirations became very rapid, irregular and gasping. At $5 \mathrm{p} . \mathrm{m}$. there was complete flaccid paralysis of the right extremities in addition to the abductor weakness now more marked in the left hind leg. No reflexes were obtained. The animal died of respiratory failure at 5:10 p. m., the heart continuing to beat for 3 minutes.

The viscera were negative except for some small subdural hemorrhages in the insertions of the muscles about the knees and hips, probably traumatic. There was marked extradural hemorrhage, edema in the first and second cervical segments and about the medulla, and subdural hemorrhage on the posterior aspect of the second cervical and third dorsal segments. The brain was generally edematous, and there was hemorrhage of the sheath of the left optic nerve, symmetrical hemorrhage at the highest portions of the cerebellar hemispheres, and smaller hemorrhages in the other portions of the cerebellum and pons. Smears of the surface of the brain-substance showed many tiny gram-positive diplococci. The blood-agar plate cultures from the blood were sterile; those from the spinal fluid and brain substance showed a great many fine, dry, green colonies of streptococci in pure culture. Microscopic sections showed hemorrhagic, infiltrative and disseminated meningomyelo-encephalitis extending throughout the brain and cord, more marked in the cerebellar, cervical and lum. bar regions. There was diffuse nerve-cell degeneration and neurophagocytosis. The infiltrating cells were mononuclear (Fig. 17). Streptococci were demonstrated in the lesions in large numbers in the depth of the tissues, while in the meninges a moderate number of leukocytes were found. 
Rabbit 999, weight $500 \mathrm{gm}$., was injected Aug. 30 and 31, 1916, with $3 \mathrm{c} \mathrm{c}$ and $5 \mathrm{c}$ c, respectively, of the culture from the brain of Rabbit 978 , which was injected intracerebrally with an emulsion of the brain of Case 714. Sept. 2, there was marked ataxia, the animal could not walk and when standing swayed as though dizzy and fell repeatedly to the left. It was excitable, had a coarse tremor of the head and forelegs, and a coarse lateral nystagmus with the quick component to the right. There was marked weakness of the extensors of the left hind leg; and greatly exaggerated knee jerks. The right side was spastic with frequent coarse jerks of the legs and at times a slight retraction of the head. The following day there was almost complete flaccid paralysis of both hind legs, marked weakness of the right foreleg, frequent general convulsions, and retraction of the head between spasms, coarse twitchings of the right front, and to a less degree, of the right hind leg. The nystagmus was still present, the knee jerks were absent. It was able to keep upright on its forelegs spread widely apart; the hind legs remained stretched out in complete extension. The ataxia had disappeared except for a slight unsteadiness of the head. Food was taken, but an attempt to eat solid food (carrot) brought on convulsions (Fig. 11). Sept. 4 , the animal was found dead.

There were marked hemorrhage and edema surrounding the dura, especially in the lumbar and cervical regions, marked congestion of the pia, subpial hemorrhages near the median fissure in the right hemisphere, hemorrhage in the pia over the anterior surface of the medulla and pons, and one large hemorrhage in the substance of the pons and medulla near the median line. A slight arthritis of the left knee, but no lesions of the viscera except one hemorrhage in the pyloric end of the stomach were found. Cultures of the blood were sterile, while those from the lumbar cord and brain showed pure growths of fine dry, green colonies of streptococci. Microscopic sections showed infiltrative mononuclear meningitis with extension along the vascular adventitia into the adjacent nerve tissue, focal hemorrhages, round-cell infiltration and nerve-cell degeneration, particularly in the gray matter of the cord. Streptococci were numerous in the pia but.were difficult to demonstrate in the nerve tissue.

Rabbit 1001, weight $450 \mathrm{gm}$., was injected Aug. 30 and 31, 1916, with the growth from $7.5 \mathrm{c} \mathrm{c}$ and $15 \mathrm{c}$ c,respectively, of the culture from the brain of Rabbit 978 . Sept. 3, the animal appeared sick and coughed; there was no lameness. The following day there was undoubted weakness in the front legs, more marked in the right than in the left. Sept. 6, the hind legs were rather clumsy and the knee jerks appeared exaggerated. Sept. 12, it was found dead.

No lesions were found except hyperemia of the vessels of the pia and a few hemorrhages in the gallbladder. Cultures from the blood were sterile; those from the brain showed a great many fine dry, green colonies of streptococci in pure culture. Microscopic sections of the brain, medulla and cord showed hyperemia and hemorrhages, in which streptococci of varying size were numerous. There were circumscribed areas of round-cell infiltration, often surrounding the blood vessels (Figs. 15 and 16). It was extremely difficult to find streptococci in the areas of round-cell infiltration. In some instances the disseminated areas of infiltration were more widespread and tended to be confluent, giving a picture of infiltration not unlike that in acute poliomyelitis in man and monkey.

Rabbit 1012, weight $330 \mathrm{gm}$., was injected Sept. 4, 1916, with the growth from $15 \mathrm{c} \mathrm{c}$ of the broth culture from a single colony of the tonsil emulsion in Case 722 . Sept. 6, it was found dead.

There were marked softening of the brain and cord, marked congestion of the vessels of the pia and extradural plexus, and hemorrhages surrounding the cord. Cultures of blood and brain showed pure growths of fine, dry, green 
colonies of streptococci, about ten times as many in the brain as in the blood. Microscopic sections showed beginning meningitis with numerous streptococci in the pia, marked degeneration in the nerve cells in the cord, and practically no cellular infiltration.

Rabbit 1017, weight $220 \mathrm{gm}$., was injected Sept. 5, 1916, with the growth from 15 c c of culture from the brain of Rabbit 999 . Sept. 7, the animal was very unsteady and weak; there was constant tremor of the head and it was just able to stand. On the following day the power in the muscles of the front legs and the left side of the neck was gone. It could pull the hind legs under it, but the front legs were spread widely, and it could not bring the thorax off the floor (Fig 12). There was no nystagmus and no reflexes were obtained. It died at $9: 30$ a. $\mathrm{m}$.

Cultures from the blood showed a few, from the brain innumerable, numbers of fine, dry, green colonies of streptococci; in those from the kidney there were many green colonies of streptococci, while in those from the liver and joints there was no growth. Microscopic sections showed diffuse hemorrhagic infiltrative meningitis extending along the central canal, infiltrative disseminations and hemorrhages, more marked in the brain and medulla, but progressively less marked in the spinal cord. Infiltration was predominatingly mononuclear and was especially noticeable in the pia and around the central canal (Fig. 13). Diplococci were found in the lesions in brain, spinal cord and meninges. They were most numerous surrounding blood vessels in the meninges and difficult to demonstrate in the areas of round-cell infiltration in the cord.

Rabbit 1032, weight $400 \mathrm{gm}$., was injected. Sept. 12, 1916, with the growth from $15 \mathrm{c} \mathrm{c}$ of the culture from the brain of Monkey 10 which was injected with culture from the brain of Rabbit 1017. This strain from Case 714 was in the fifth animal passage. Sept. 14, the animal was found dead.

There were a few hemorrhages in the right knee joint, and the joint fluid was turbid. There were hemorrhage and infiltration of the muscles of the anterior surface of both tibias. There were numerous, very small punctate hemorrhages in the subcutaneous tissue, marked edema and hemorrhage in the lungs, with beginning bronchopneumonia, many small hemorrhages in the kidneys, and cloudy swelling of the viscera. There was hemorrhagic edema surrounding the cerebellum. The cerebrospinal fluid was turbid. There were localized hemorrhages under the dura in the lumbar region, and edema surrounding the cord and dura along the nerve roots and ganglia. Blood-agar plate culture from the blood and spinal fluid were negative. Those from the kidney showed countless fine, dry, green streptococci and those from the liver a moderate number of similar colonies. Microscopic sections revealed a large hemorrhage in the left cerebellar hemisphere and a moderate number of small hemorrhages in the medulla and cord, which gave the picture of a mild meningomyelitis. The infiltrating cells in the pia consisted of about equal numbers of round cells and leukocytes. Streptococci were present in enormous numbers surrounding blood vessels, especially in the pia and the more superficial vessels in the cord, and in localized areas showing hemorrhage or degeneration of nerve cells in the gray matter of the medulla and cord.

Dog 463, weight $4.5 \mathrm{~kg}$., was injected Aug. 4, 1916, with the growth from $45 \mathrm{c} \mathrm{c}$ of broth culture from the tonsil of Case 695 . On the following day at noon the animal was lame in the right hind leg (the injected leg). There was tremor in both hind legs, and apparently definite weakness. At $6 \mathrm{p}$. $\mathrm{m}$. the muscular tremor had extended to the right front leg, and at 9 p. m. it was unable to stand, and lay on its side with head retracted and extremities held stiff. There was no swelling of the joints. Aug. 6, it was found dead. 
There was marked cellulitis at the point of injection. There were hemorrhages in the lungs, gallbladder and liver, and marked congestion of the vessels of the cord and brain. The meningeal fluid was turbid, the pia over the brain was opaque, and the brain was edematous and soft. There were hemorrhagic spots in the anterior horns of the cervical and lumbar cords and smears from the meningeal fluid showed a few gram-positive diplococci. Cultures from the meningeal fluid and blood showed what appeared to be pure culture of pneumococci. Microscopic sections showed extensive meningitis and disseminated areas of infiltrative and perivascular infiltration principally with mononuclear cells. Hemorrhages and diffuse degenerative lesions were present in the brain and spinal cord and ganglia. Streptococci were demonstrated in the hemorrhagic areas and in the meninges in moderate numbers, and in smaller numbers in the areas of hemorrhage and round-cell infiltration in the substance of the cord.

Cat 11, weight $930 \mathrm{gm}$., was injected, Aug. 24, with the growth from $45 \mathrm{c}$ c of tonsil pus from Case 712. Aug. 26, there was weakness in the hind legs, most marked on the right. The temperature was $104 \mathrm{~F}$. Sept. 11, the animal was found dead; weight $690 \mathrm{gm}$.

I

II III

IV

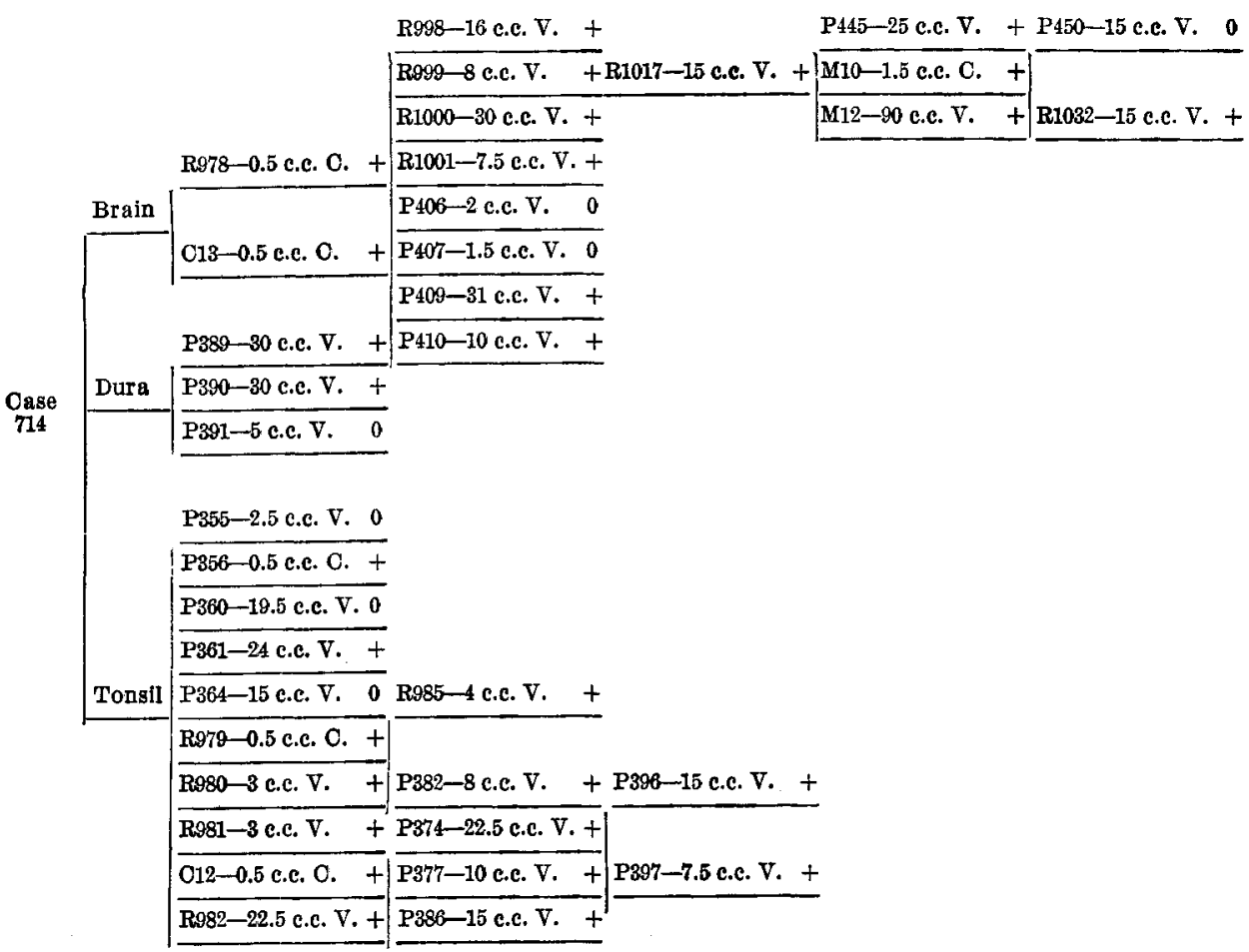

Text Fig. 1.-Chart showing results of injections of emulsions and cultures of the pleomorphic streptococeus from brain, edema fluid surrounding dura, and tonsil in a typical case of poliomyelitis before and after animal passage. $V$, intravenous inoculation; $C$, intracerebral inoculation; + , positive result; 0, negative result. All intracerebral injections were emulsions of brain or tonsil. All intravenous injections consisted of cultures or suspensions of cultures. 


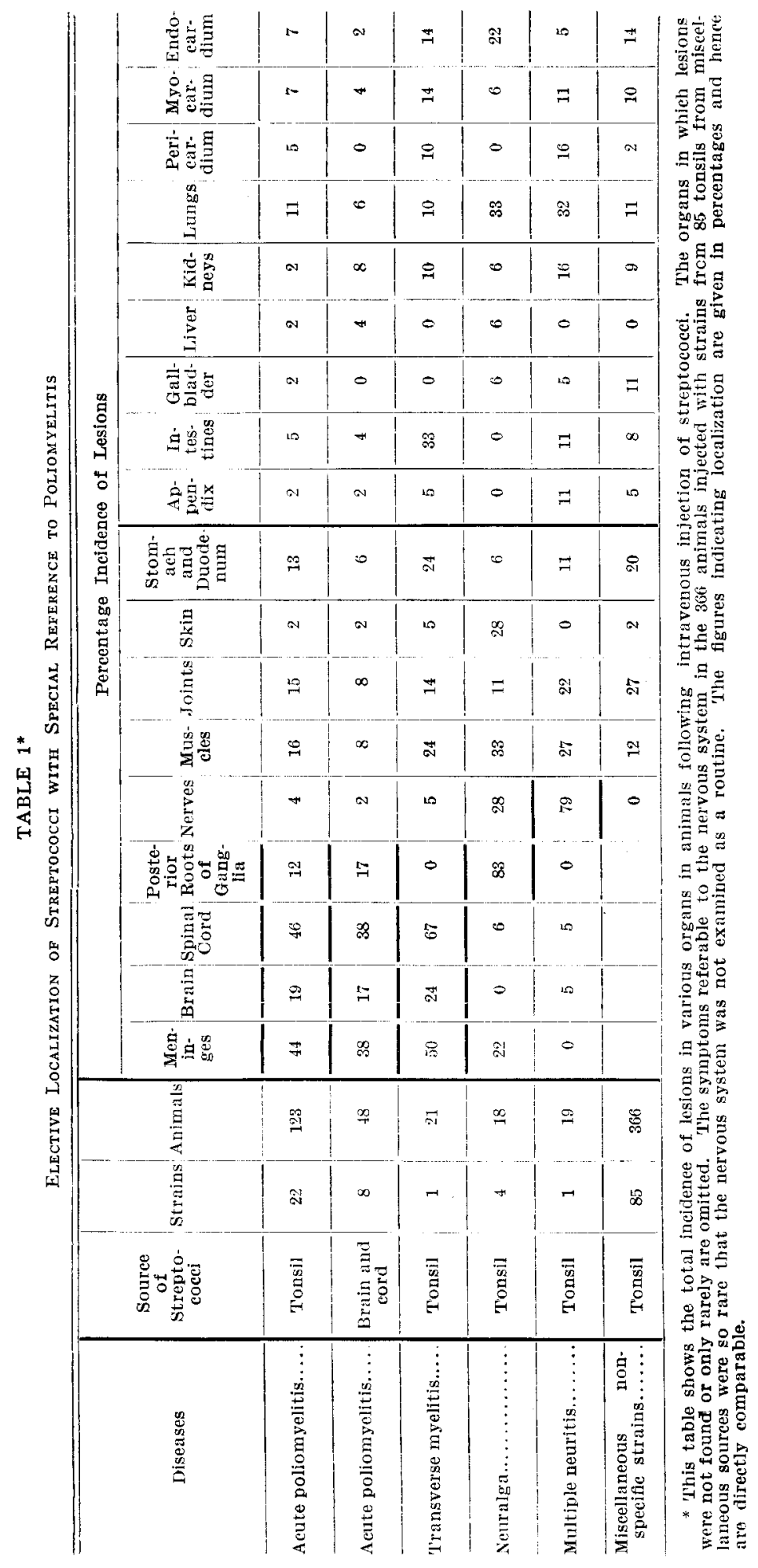


There were no lesions of the viscera but edema and cloudiness of the meninges and a large amount of turbid cerebrospinal fluid. Blood-agar plate cultures from the kidney, liver and blood were negative, those from the spinal fluid, brain and lumbar cord contained countless numbers of fine, dry, green colonies of streptococci. Sections of the central nervous system showed definite mixed-cell meningitis degenerations and hemorrhages in the brain and cord, and streptococci.

\section{EXPERIMENTS ON INTRACEREBRAL INCCULATIONS}

It was thought advisable to compare the results of intracerebral injections with intravenous injections. Hence, young guinea-pigs, rabbits and cats were injected intracerebrally with from $0.25 \mathrm{c} \mathrm{c}-1 \mathrm{c} \mathrm{c}$ of suspensions of pus from tonsils, and of emulsions of brain from human poliomyelitis and from animals showing paralysis, and filtrates of emulsions and cultures.

Emulsions from the tonsil of 6 cases of human poliomyelitis were injected altogether into 3 rabbits, 5 guinea-pigs and 4 kittens. Seven of the 12 animals developed flaccid paralysis usually complicated with symptoms of meningitis. In 9 the blood was sterile or nearly sterile, while the brain and spinal fluid contained many streptococci. In all, the green-producing streptococcus predominated, and in all but three instances it was isolated in pure culture (Rabbit 929 and Guinea-pig 433). Meningitis was usually the cause of death and the chief finding at necropsy. Recovery occurred in some instances (Rabbit 929).

Emulsions of the brain and cord from 6 human cases and from 4 animals showing flaccid paralysis were injected into 14 guinea pigs, 11 rabbits and 4 kittens. Flaccid paralysis developed in 8 animals; in 7 the pleomorphic streptococcus was isolated in pure culture from the nervous system, usually in large numbers, but not from the blood (Rabbits 978 and 1018). The other animals showed no symptoms. Ten died within 24 hours, most of them apparently from mechanical and toxic effects, the cultures being negative. Fourteen recovered completely, at no time showing symptoms. Five were chloroformed or died from 2-6 days after injection. The emulsions of the brain and cord from 3 human cases gave positive results, while the remaining 3 gave negative results.

Owing to the marked variations in size of the streptococcus having affinity for the nervous system, some forms being fully as small as the globoid bodies, filtrates of emulsion and cultures were used for injection. The first filtrate was cultured and injected Aug. 26, 1916. Berkefeld filter $\mathrm{N}$ Berkefeld filter $\mathrm{V}$, and a dense porcelain candle were used. The latter is the type of filter which was used as a control in elective localization studies. Altogether 8 filtrates were made from emulsions 
of brain and cord of animals showing flaccid paralysis. These were injected into 11 guinea-pigs, 12 rabbits and 2 kittens. Eight of the animals died within 24 hours, usually without paralytic symptoms, the blood and brain being sterile. Four died within 4 days, 3 showing streptococci in the brain but not in the blood. Thirteen remained well and were anesthetized a long time after injection.

In three instances parallel filtrates were made through a Berkefeld $\mathrm{N}$ and porcelain filters. Cultures and injections of the latter were negative. Two of the former yielded the pleomorphic streptococcus in pure form on culture. Injection of one of these was followed by paralytic symptoms (Rabbit 1023), the other rabbit showed streptococci in the brain but not in the blood. One other Berkefeld filtrate gave positive results in a rabbit but the cultures remained sterile. Four Berkefeld $N$. filtrates were sterile and the results in animals were entirely negative. The lesions observed in these animals following intracerebral injection consisted chiefly of a meningitis with more or less infiltration and degeneration of the nerve cells in the brain and cord. In the animals that died early the meningitis was chiefly polynuclear; in the animals that lived for some time is was mononuclear. The infiltration was usually most marked around the blood vessels, around the central canal and in the anterior horns. Degeneration of the nerve cells in the medulla and anterior horns, more especially of the cervical cord, was noted. These lesions following intracerebral inoculation resembled very closely those described by Rosenau and Havens following successive injections of virus in rabbits.

\section{Illustrative Protocols Following Intracerebral Inoculation}

Rabbit 929, weight $660 \mathrm{gm}$., was injected July 21, 1916, with $1 \mathrm{c} \mathrm{c}$ of the emulsion in salt solution of the pus in Case 686. July 22, it appeared quite well, but was spastic and the knee jerks were exaggerated. July 24 , it stood with head markedly retracted. The eyes were more prominent than normal. When made to walk it appeared awkward and unsteady and the right hind leg was thrown out. It became tired in a few minutes and the right hind leg was markedly weak, gave way and dragged. The right foreleg also was weak. The rabbit tended to fall to the right. The muscle tone in the hind legs and right front leg was low. Spasticity had disappeared and no reflexes could be obtained. July 25 , it was lame in the left hind leg, the knee joint was swollen, but the right leg appeared stronger. Aug. 12, it had apparently regained full power in the extremities and appeared well. It was chloroformed.

The blood cultures were sterile, those from the brain yielded a pure culture of a streptococcus varying matkedly in size, some forms resembling "globoids."

Guinea-pig 433, weight $200 \mathrm{gm}$., was injected Sept. 6, 1916, at 7:30 p. m. with $0.25 \mathrm{c} \mathrm{c}$ of the emulsion of the tonsil from Case 724 . At $10 \mathrm{p} . \mathrm{m}$. there was a fine tremor all over the body. It was excitable, acted strangely, pushed its head violently against the floor of the cage. There was somfe difficulty in walking. the hind legs tending to extend, and the tremor was much worse on attempts to 
walk. At 10:20 p. m. it was found pushing violently with its nose against the dish containing oats. When freed from this position it ran its nose forcibly into the side of the cage and attempted constantly to bury it deeply in the hay. Sept. 7 , it was found dead.

There was edema of the lungs, marked hemorrhage surrounding the dura, especially in the cervical region and cerebellum and medulla. There was no hemorrhage at the point of injection. Cultures from the blood were sterile, while those from the blood and brain showed the pleomorphic streptococcus in pure culture.

Rabbit 978, weight $480 \mathrm{gm}$., was injected Aug. 24, 1916, with $0.75 \mathrm{c} \mathrm{c}$ of the emulsion of the brain of Case 714 (Fig. 1). Aug. 27, there was no loss of power of the extremities, but the animal acted peculiarly with a tendency to lie down and stretch out the hind legs after it had been made to walk. Aug. 28 at noon, there was very marked general weakness. The extensors of the left foreleg were completely gone; those of the right were weak. The neck muscles were very weak; the head was not held up and usually fell to the left. The hind legs were generally weak, the right more marked, but it could pull them under it when extended. The respirations were slow and shallow; no motion of the ribs could be made out. It died at $1 \mathrm{p} . \mathrm{m}$.

There were superficial recent hemorrhages in the mucous membrane of the stomach, and hyperemia and hemorrhage surrounding the dura in the cervical region. The brain showed no gross lesions. Cultures from the blood were sterile, those from the brain showed the pleomorphic streptococcus in large numbers. Microscopic sections showed the picture of a leptomeningo-encephalitis, the predominating infiltrating cell being mononuclear.

Rabbit 1018, weight $580 \mathrm{gm}$., was injected Sept. 6, 1916, with $0.5 \mathrm{cc}$ of the emulsion of the brain in Case 724. Sept. 11 there was double foot drop behind. It could walk a few steps very awkwardly, dragging the toes. The legs spread apart as if there was adductor weakness. When the hind legs were pulled out back it got them under by pushing the body back with the front legs. The animal appeared in good general condition. Sept. 12, it moved about much better, though the left hind leg was undoubtedly weak. Sept. 15, it was found dead.

There was marked edema surrounding the dura of the cord; no apparent lesion at the point of injection. There were marked congestion of the vessels of the meninges, edema surrounding the medulla and cerebellum and no lesions of the viscera. Cultures from the blood were negative, those from the brain showed countless numbers of green colonies of streptococci resembling pneumococci. There were hemorrhagic meningitis, marked round-cell infiltration of the choroid plexus and localized areas of round-cell infiltration around the vessels of the brain, pons, and medulla (Fig. 19).

Rabbit 993,* weight $610 \mathrm{gm}$., was injected Aug. 30, 1916, with filtrate of ascites fluid culture of the brain of Rabbit 978. Aug. 31, the animal was found dead.

There were marked degeneration of the liver, cloudy swelling of the viscera, no hemorrhage at the point of injection, and edema of the brain and cord surrounding the dura. Cultures from the blood were sterile, while those from the brain yielded pure culture of the pleomorphic streptococcus.

Rabbit 1023, weight $670 \mathrm{gm}$., was injected Sept. 8, 1916, with $0.5 \mathrm{cc}$ of a Berkefeld $N$ filtrate of the emulsion of the brain of Rabbit 1017. Sept. 10, the head was markedly retracted, the animal moved constantly, could stand but was very ataxic when it attempted to walk. The hind legs were spread

* The cultures of the filtrate injected were negative on blood agar but showed the pleomorphic streptococcus in deep cultures of ascites-dextrose tissue broth. 
apart and there was marked adductor weakness, especially of the right leg. Respirations were rapid. Sept. 11, it was found dead.

The body was warm. There were numerous hemorrhages in the stomach and a few areas of infection in the left kidney; marked edema over the brain, turbid cerebrospinal fluid, and hemorrhages of the posterior aspect of the cerebellum. Cross sections of the medulla showed a number of hemorrhages in the gray matter and some hemorrhages in the cervical cord. Blood-agar plate cultures of the blood showed a few colon bacilli, but no streptococci. Cultures of the brain and lumbar cord showed countless numbers of the fine dry green colonies in pure culture, while those from the liver and kidney were negative.

\section{SYMPTOMS}

The symptoms observed varied greatly but were usually striking and easily differentiated from arthritis, myositis and from general weakness occurring in overwhelming infections. In many instances the animals appeared well, ate food normally, were active and seemed disturbed because of inability to use the paralyzed part. There were usually instability, restlessness and evidences of pain on handling, especially in the guinea-pigs (Pig 346). Tremor and twitchings of muscles of one or more extremities often preceded the development of flaccid paralysis (Pig 264). In some instances these premonitory symptoms were followed by recovery without development of paralysis. Increased reflexes followed by loss of reflexes and loss of muscle-tone were common findings. The paralytic symptoms varied from slight weakness, usually in extensor groups, to complete flaccid paralysis of one or more extremities.

Death from respiratory failure was the rule in the animals observed (Pigs 262 and 264, and Rabbits 981, 982, 985, etc.). Some died in convulsions. This was especially apt to occur when paralysis began in the fore extremities. The place of onset of the paralysis in some animals corresponded to that in the patient from whom the culture was obtained. This occurred too often to be accidental.

Flaccid paralysis was the striking symptom in many animals, but this was often associated early with more or less evidence of meningeal irritation (Pigs 262, 264, 266, 270, 352, 374, 384, 396, and Rabbits 962 , $977,980,981,982,1001,1017)$. In others the paralysis appeared to be associated with a definite meningitis (Pigs 234, 422, 430, Rabbit 985, and Dog 463). In a few cerebellar symptoms predominated (Rabbit 999). A number of guinea-pigs and rabbits and one cat showed symptoms suggesting mental derangement (Pigs 422, 430, 433, and Rabbit 985). The time of onset of symptoms was related both to the size of the dose and to the infecting qualities of the particular strain injected. Thus, in some instances the symptoms began late if the dose was small 
(Rabbits 980 and 981) and early if the dose was large (Rabbit 982), while in others they began simultaneously and ran the same course even though the dose varied by the fifteenth multiple (Pigs 266 and 270 ). In some instances paralytic symptoms began as early as 4 hours, and often within 24 hours, following inoculation (Pigs 264, 266, 270, 324, and Rabbits 962, 977 and 985). The symptoms were prone to be of an explosive character when the period of incubation was long (Rabbits 980 and 981). This finding is in accord with a similar observation made by Rosenau and Havens following injection of virus.

\section{GROSS AND MICROSCOPIC CHANGES}

The gross pathologic findings consisted of hyperemia of the vessels of the meninges and an increased amount of cerebrospinal fluid, usually clear, sometimes slightly turbid, and in a few instances markedly turbid. The anterior extradural plexus, particularly at the lumbar and cervical enlargements of the cord, showed extreme engorgement. The anterior surface of the dura was often hemorrhagic and edematous. Hemorrhage and edema around nerve-roots and spinal ganglia were a common finding. The pia in some instances appeared opaque but was usually moist and slimy. Hyperemia, edema and focal hemorrhages in the gray matter of brain, medulla and cord, chiefly in the anterior horns, were frequently noted.

The lymph glands, particularly those in the mesentery and retroperitoneum, were often swollen, edematous and hemorrhagic. The lymphoid elements in the intestines at times appeared swollen. There was often marked edema in the retroperitoneum in and about the solar plexus and pancreas in guinea-pigs (Pigs 266, 270, 324, and 337). This has never been seen following intravenous injection of streptococci from sources other than poliomyelitis. The spleen rarely showed enlargement. The lesions in other organs were conspicuously rare and when they did occur were relatively slight.

Microscopically, the meninges and nerve tissue showed a variable vascular reaction ranging from slight hyperemia to marked engorgement. In many cases this was accompanied by perivascular and interstitial hemorrhages, more marked in the gray matter. Infiltration with small round cells (Fig. 13) occurred in the pial membrane and in the adventitia about the blood vessels (Figs. 6, 15, 16, and 19). In other cases the infiltration was diffuse over wide areas in the interstitial tissues, or more often in circumscribed areas either around a small vascular focus or circumscribing a central point of cellular degeneration 
(Figs. 13, 14, 17, and 19). In places these islands tended to be confluent. Generally the lesions varied from the larger and more discrete areas to the smaller and more confluent areas resembling the finely disseminated coalescing lesions in typical poliomyelitis. Where they were more extensive a central focus of hemorrhage, or, more often, an area of dense cellular infiltration with disintegrated stroma was surrounded by a wide zone of mononuclear infiltration both about the blood vessels and in the intervening tissues. A section through the peripheral zone of such an area gave a picture microscopically not unlike that in the diffuse infiltration of an acute poliomyelitis in man. Until serial sections were studied to determine the presence or absence of a central focus, such an infiltration could not be accurately interpreted. Some animals showed adventitial tissue and diffuse interstitial cellular infiltration with no evident central focus (Figs 13 and 14). In a few animals thrombosis of the vessels of the meninges and cord were found with little or no perivascular infiltration.

Cytologists do not yet agree regarding the origin of the infiltrating round cells, hence no special attempt was made to identify them. Various types resembling lymphocytes, proliferated endothelial cells and glial cells took part in the reaction. Leukocytic infiltration was relatively slight. Even in animals showing meningitis the infiltration was usually predominatingly mononuclear. The infiltrative lesions in the depths of the brain and cord at times contained only mononuclear cells when meninges showed both (Rabbits 985 and 1017, Fig. 13). Leukocytic infiltration was more often prominent in experiments of short duration following injection of large doses (Rabbits 980, 981 and 982), and particularly after injection of these strains rendered more virulent by repeated animal passage (Rabbit 1032). The nerve cells often showed swelling, granular staining, and in advanced stages they stained poorly or not at all. In many instances small round cells, sometimes recognized as glial cells, collected around degenerated cells as satellites, but marked neurophagocytosis was not common.

Streptococci were found in or adjacent to the lesions in the central nervous system in all animals that showed symptoms not too long preceding the time of death. In the animals in which the experiment was of short duration and the lesions were acute, the demonstration of streptococci was relatively easy. The streptococci here were most numerous in the perivascular lymph spaces and other areas where infiltrative lesions more often became marked in later stages. In the perivascular areas they were of uniform size, resembling pneumococci, while in the infiltrative areas great variation in size were 
noted, some diplococi being fully as small as the "globoid" organism described by Flexner and Noguchi. Streptococci were found in the edematous and hemorrhagic areas surrounding the dura, in the pia, in the adventitial lymph spaces surrounding dilated blood vessels (Fig 9), in edematous and hemorrhagic areas in the brain and cord (Figs. 8, 18 , and 20) and in areas of perivascular and interstitial round-cell infiltrations (Fig. 7). In the latter it is often exceedingly difficult to find bacteria. In a few animals that had recovered completely from paralysis, streptococci could not be demonstrated. The tissues showing no lesions were quite free from streptococci even in experiments of short duration and where streptococci were demonstrable in the lesions that were present.

\section{THE RESULTS}

The results of the injection of cultures from the tonsils or throat of 22 cases and those from the brain and cord in 8 cases were studied in detail.*

Altogether 171 animals were injected intravenously; young guineapigs, rabbits, dogs, cats, and monkeys; 123 were injected with tonsil strains and 48 with brain and cord strains. The tonsil strains were injected into 75 guinea-pigs, 36 rabbits, 10 dogs, 1 cat, and 1 monkey; the brain and cord strains into 25 guinea-pigs, 19 rabbits, 1 dog, 1 cat, and 2 monkeys.

Positive results were obtained with cultures from tonsils made on repeated occasions, in one case on four occasions, and with cultures from different parts of the central nervous system (Text Fig. 1). In one instance completely negative results followed the injection of a pneumococcus from the consolidated lung when the streptococcus from the tonsil and the brain and cord from the same patient produced paralysis. The affinity for the central nervous system at the time of injection varied greatly in different strains. Approximately $70 \%$ of the animals were inoculated with the first culture generation, $25 \%$ with the second, and $5 \%$ with the third or fourth. When platings were made, subcultures for injection were made as soon as colonies became recognizable. Twenty-five per cent. of the rabbits weighed less than $500 \mathrm{gm}$., $55 \%$ between 500 and $1,000 \mathrm{gm}$., and only $20 \%$ above $1,000 \mathrm{gm}$. Fifty per cent of the guinea-pigs weighed less than $200 \mathrm{gm} ., 40 \%$ between 200 and $300 \mathrm{gm}$., and only $10 \%$ above $300 \mathrm{gm}$. The average weight of guinea-pigs showing positive results was about $200 \mathrm{gm}$., of rabbits about $600 \mathrm{gm}$. The average weight of guinea-pigs

\footnotetext{
* For methods and results of cultures see Reference 48 and the paper in this series on the etiology of poliomyelitis.
} 
and rabbits with negative results was about $300 \mathrm{gm}$. and 1,200 gm., respectively.

The diagnosis of localization in the central nervous system was based in the order of importance on four main findings: (1) Microscopic lesions of the central nervous system; (2) streptococci in or adjacent to these lesions; (3) positive cultures from the central nervous system when blood or other tissues were sterile or when they contained comparatively few bacteria, and (4) meningitic or paralytic symptoms.

In $40 \%$ of the animals, paralytic symptoms were noted during life. In approximately $70 \%$ of the animals in both series two or three of the diagnostic factors were present, leaving no reasonable doubt of the specific localization. In $15 \%$ following injection of strains from tonsils, and in 20\% following injection of strains from brain and spinal cord, all the diagnostic factors were present and the specific localization proved beyond doubt.

Seventy-three of the 171 animals were chloroformed for examination after definite symptoms were established; 98 died. The mortality rate in rabbits was considerably higher than that in guinea-pigs. In both species it was somewhat higher following the injection of tonsil strains than with brain and cord strains. The total percentage incidence of lesions in various organs following injection of the poliomyelitis strains together with that of a number of other strains is given in Table 1. The tendency of the strains from poliomyelitis and transverse myelitis to produce lesions in the central nervous system was striking. The occurrence of lesions in other organs was comparatively rare following injection of the poliomyelitis strains, but occurred more often following injection of tonsil strains than following the injection of brain and cord strains. Lesions outside the central nervous system following injection of cultures from tonsils were found to be due in some instances to nonspecific strains of streptococci or staphylococci (Rabbits 962 and 982). Unless the specific affinity was marked on isolation, aerobic cultivation for several generations was sufficient to destroy the elective localizing power. Small doses after aerobic cultivation produced no lesions whatever; large doses produced slight lesions in stomach, kidney or joints with or without focal lesions in the central nervous system. Moreover, on repeated animal passages, even when cultures for succeeding injections were made from the brain or spinal fluid, the specific localizing power gradually disappeared. The range of localization and the degree of lesions became greater as virulence increased. Hemorrhages and edema of the lung, hemor- 
rhages of stomach, suppurative arthritis and suppurative focal lesions in the brain and cord, and purulent meningitis were now more likely to occur (Rabbit 1032).

The incidence of localization before and after successive animal passages of the strain from the brain, from the edematous fluid surrounding the dura, and from the tonsil of Case 714, is illustrated in text Figure 1. Sixteen animals were injected intravenously with brain and dura strains and fourteen with the tonsil strain. Of the former twelve $(75 \%)$, of the latter eleven $(71 \%)$, showed undoubted symptoms or symptoms referable to lesions of the central nervous system. Some of the negative results were apparently due to the smallness of the dose (Pigs 391, 406, 407 and 955). Positive results were obtained when the dose varied from the second to the seventh multiple (Rabbits 998, 999, 980, 981 and 982). The strains isolated from many of these animals were cross-agglutinated specifically by the serum from a horse immunized with human strains and another horse immunized with monkey strains. ${ }^{4 T}$ The serum from persons and monkeys which had recovered from poliomyelitic attacks had a similar but less marked agglutinating power. ${ }^{4 \pi}$

Cultures from the brain and cord, and blood of the animals were made routinely and from various other tissues in many instances. In most animals showing positive symptoms the brain and cord showed pure cultures of streptococcus, often in large numbers when the blood and other tissues (with the exception of the kidney) contained few or none (Pigs 264, 266, 270, 324, 352, Rabbits 962, 985, 999, 1001, 1012, and 1017). The importance of using partial oxygen-tension cultures (tall columns of dextrose or ascites-dextrose broth) in this work, aside from differences in the mediums, was shown by the fact that aerobic blood-agar plates from brain and cord usually remained sterile when the deep cultures in the broth were positive (Rabbits 980 and 981) unless the number of bacteria was large, then both gave positive results.

The number of streptococci isolated from brain and spinal fluid was, as a rule, definitely related to the duration of the experiment and sometimes to the symptoms referable to the central nervous system. This is in accord with the microscopic findings. The blood of the animals injected with the strains, as isolated, almost never showed large numbers of streptococci even in those that died within 24 or 48 hours after inoculation.

Control cultures, using the same technic, from brain and spinal fluid and blood were made in 35 animals (29 guinea-pigs, 4 rabbits 
and 2 dogs) which were killed some months after inoculation. These had been injected either with small doses of the characteristic streptococcus, with hemolyzing streptococci from tonsils or with mixtures of bacteria from tonsils some time after recovery from the attack. In these streptococci were not isolated in a single instance. Cultures of tissues were made, moreover, in 40 uninoculated animals as controls; 33 of these were well and were killed for examination, and 7 died from unknown cause. One of the former and three of the latter showed a few colonies of green-producing streptococci in small numbers in the cultures from brain and spinal fluid, and in two of the latter indifferent streptococci were isolated from the blood and brain. Colon bacilli were isolated from blood and brain in a few that had died, but not from those killed immediately before examination.

\section{DISCUSSION}

Following the discovery that the virus of poliomyelitis is filtrable, ordinary bacteria were considered of little or no etiologic importance in this disease, until the demonstration recently that the tonsils and infected tissues in acute poliomyelitis contained streptococci which, when injected into animals, tended to localize in the central nervous system. Bull studied streptococci from tonsils in poliomyelitis and from the tonsils and teeth of persons who were not suffering from poliomyelitis, nor in immediate contact with the disease, but who resided in the midst of a well-spread epidemic. It is to be noted that his technic, although purported to be done "in the manner described by these authors," really does not fulfill the conditions emphasized as essential in the study of elective localization in poliomyelitis. Young animals must be selected. Only adult guinea-pigs and rabbits were used in Bull's experiments. Twelve guinea-pigs weighing from 450$500 \mathrm{gm}$. were injected, with negative results. In the experiments reported in this paper 100 young guinea-pigs were used with positive results in many. The average weights of those giving positive results was $200 \mathrm{gm}$.; of those with negative results $300 \mathrm{gm}$. The average weight of the rabbits as given in Bull's protocols was $1,580 \mathrm{gm}$. The average weight of our rabbits showing positive results was $600 \mathrm{gm}$., while in those showing negative results it was $1,200 \mathrm{gm}$. Bull injected massive doses of streptococci from tonsils grown aerobically at $37 \mathrm{C}$. (chiefly on ascites-dextrose agar slants). We injected varying-sized doses from tonsils and brain and cord grown at from 33-35 C. in tall tubes of ascites-dextrose broth affording a gradient of oxygen-tension. 
Only $10 \%$ of Bull's animals received the primary culture, $70 \%$ the second, and $20 \%$ the third. In contrast, $70 \%$ of the animals reported in this paper received the primary culture. $25 \%$ the second, and $5 \%$ the third or higher culture generation.

With such differences in methods one is not surprised at the differences in results. However, disregarding the fact that Bull failed to produce symptoms and lesions characteristic of poliomyelitis, localization of streptococci in the central nervous system occurred commonly even in his experiments. Thus, in rabbits, localization occurred more than half as often in the nervous system as in all other organs combined. The blood in his animals, as in ours, was often sterile when brain and meninges showed large numbers of streptococci. That the streptococci from the tonsils of persons who had no obvious symptoms of poliomyelitis, but who resided in the midst of a widespread epidemic, should show streptococci of similar virulence and localizing power is in harmony with results of epidemiologic studies which indicate more and more the widespread prevalence in unrecognized form of the infective agent of this disease.

The symptoms in many of our animals, as shown in the protocols, were quite like those observed in poliomyelitis in man. They were chiefly meningitic in the beginning and later paralytic. Twitchings and spasms of muscles, hyperesthesia and irritability often preceded the onset of paralysis. Death from respiratory failure was common, especially if paralysis began in the anterior extremities. In guinea-pigs improvement in muscle function, with or without contractures, occurred more commonly than in rabbits. In the latter a well established paralysis usually progressed until death. Lesions in other organs were comparatively uncommon (Table 1). The lesions resembled in type those present in poliomyelitis in man and monkey, although in distribution they were not wholly identical. Results comparable to these have not been obtained by any one following inoculations of streptococci from a wide range of sources other than from poliomyelitis or other diseases of the nervous system. That the symptoms and lesions in rabbits must in every way be identical to those in poliomyelitis in man and monkey to be of significance, is open to question. Marks succeeded in passing virus which was adapted to the monkey through a series of six rabbits, producing atypical symptoms and lesions. He was then again able to produce typical symptoms and lesions in the monkey, thus proving that the virus was not modified and that the atypical symptoms and lesions in the rabbit must be 
regarded as equivalent to poliomyelitis. The disease induced in rabbits by Marks, and by Rosenau and Havens following inoculation of virus, in guinea-pigs and rabbits by us, and in rabbits by Mathers, and Nuzum and Herzog following inoculation of the pleomorphic streptococcus which is much alike, may therefore be considered as poliomyelitis in these species of animals.

\section{CONCLUSIONS}

The streptococcus found so constantly in poliomyelitis tends to localize electively in the central nervous system in young guinea-pigs, rabbits, puppies and kittens, and less often in adult animals of these species and in adult monkeys. The symptoms and lesions produced resemble those found in poliomyelitis in man.

Cultivation on artificial media, especially under aerobic conditions, usually destroys promptly the elective localizing power, as does successive animal passage. The results obtained indicate strongly that the streptococcus under consideration is not merely a secondary invader, but that it has etiologic significance in poliomyelitis.

1. Blake, F. G.: The classification of streptococci. Jour. Med. Res., 1917, 36, 99-133.

2. Bourges, H.: Myelite aiguë experimentale produite par l'erysipelocoque. Compt. rend. Soc. de biol., 1893, 9, s. 5, 184-187.

3. Bülow-Hanson and Harbitz, F.: Beitrag zur Lehre der acuten Poliomyelitis. Beitr. z. path. Anat. u. z. allg. Path., 1899, 25, 517-546.

4. Bull, C. G.: The pathologic effects of streptococci from cases of poliomyelitis and other sources. Jour. Exper. Med., 1917, 25, 557-580.

5. Buzzard, E. F.: On certain acute infective or toxic conditions of the nervous system. Brain, 1907, 30, 1-100, p. 23.

6. Concetti, L.: Ueber Eklampsie, Tetanie und Poliomyelencephalitis der Kinder. Allg. Wien. med. Ztg., 1900, 45, 279-280; 291-292; 313-314.

7. Davis, D. J.: Bacteriology and pathology of the tonsils with special reference to chronic articular, renal, and cardiac lesions. Jour. Infect. Dis., 1912, $10,148-161$.

8. Detweiler, H. K., and Robinson, W. L.: Experimental endocarditis. Its production with Streptococcus viridans of low virulence. Jour. Am. Med. Assn., 1916, 67, 1653-1658.

9. Engel, F.: Bakteriologisches Ergebnis einer Lumbalpunction bei Poliomyelitis anterior. Prag. med. Wchnschr., 1900, 25, 135-137. Cited by Römer, P. H., loc. cit.

10. Flexner, S., and Lewis, P. A.: The transmission of acute poliomyelitis to monkeys. Jour. Am. Med. Assn., 1909, 53, 1639.

11. Flexner, S., and Noguchi, H.: Experiments on the cultivation of the microörganism causing epidemic poliomyelitis. Jour. Exper. Med., 1913, 18, $461-485$. 
12. Fox, H.: Report of the epidemic of acute poliomyelitis in northwestern Pennsylvania during the autumn of 1907. Rep. Com. Health Penn., Harrisburg, 1908, 2, 420-441.

13. Fox, H., Rucker, J. B., and Rivas, D.: Report on the investigation into poliomyelitis, covering subjects of transmission, parasitology, pathology and bacteriology. Ann. Rep. Com. Health Penn., Harrisburg, 1910, 456-508.

14. Giersvold, M.: Epidemisk poliomyelit bakteriologiske undersøgelser. Norsk Mag. f. Lægevidensk., 1905, 3, 1280-1300.

15. Gerdine, L., and Helmholz, H. F.: Duodenal ulcer in infancy an infectious disease. Am. Jour. Dis. Child, 1915, 10, 397-409.

16. Hardt, L. L. J.: The secretion of gastric juice in cases of gastric and duodenal ulcers. Am. Jour. Physiol., 1916, 40, 314-331.

17. Hektoen, L.: Recent investigations on the bacteriology of acute poliomyelitis. Boston Med. and Surg. Jour., 1917, 176, 687-695.

18. Henrici, A. T.: The specificity of streptococci. Jour. Infect. Dis., 1916, 19, 572-605.

19. Hoche: Experimentelle Beiträge zur Pathologie des Rückenmarkes. Arch. f. Psychiat., 1899, 32, 209. Cited by Wickman, I., loc. cit.

20. Horder, T. J.: Infective endocarditis. With an analysis of 150 cases and with special reference to the chronic form of the disease. Quart. Jour. Med., 1908-9, 2, 289-324.

21. Irons, E. E., Brown, E. V. L., and Nadler, W. H.: The localization of streptococci in the eye. A study of experimental iridocyclitis in rabbits. Jour. Infect. Dis., 1916, 18, 315-334.

22. Kolmer, J. A., Brown, C. P., and Freese, A. M.: A contribution to the bacteriology of acute anterior poliomyelitis. Jour. Exper. Med., 1917, 25, 789-806.

23. Kolmer, J. A., and Freese, A. M.: Complement fixation in acute anterior poliomyelitis. Jour. Immunol., 1917, 2, 327-339.

24. Landsteiner, K.: Poliomyelitis acuta. In Kolle, W., and Wassermann, A.: Handbuch der pathogen Mikroorganismen. Jena, Fischer, 1913, 8, 427-462.

25. Landsteiner, K., and Popper, E.: Uebertragung der Poliomyelitis acuta auf Affen. Ztschr. f. Immunitätsforsch. u. exper. Therap., 1909, 2, 377-390.

26. Leiner, C., and Wiesner, R.: Experimentelle Untersuchungen über Poliomyelitis acuta anterior. Wien. klin. Wchnschr., 1909, 22, 1698-1701.

27. Looft and Dethloff: To Tilfälder of Poliomyelites anterior acuta has hörn Lumbalpunktion. Bacteriologisk undersögelse at spinalvälsken. Med. Rev., 1901, No. 11. Cited by Römer, P. H., loc. cit.

28. Marinesco, G.: Nature et le Traitement de la Myelite aigue. Nouv. iconog. de la Salpetriere, 1900, 13, 561-596.

29. Marks, H. K.: Infection of rabbits with the virus of poliomyelitis. Jour. Exper. Med., 1911, 14, 116-123.

30. Mathers, G.: Some bacteriologic observations on poliomyelitis. Preliminary report. Jour. Am. Med. Assn., 1916, 67, 1019.

31. Mathers, G.: The etiology of acute epidemic poliomyelitis. Jour. Infect. Dis., 1917, 20, 113-124.

32. Moody, A. M.: Lesions in rabbits produced by streptococci from chronic alveolar abscesses. Jour. Infect. Dis., 1916, 19, 515-525.

33. Morel and Rispal: Congres Francais de méd. Bord., 1895, Fasc. II (1896), p. 380. Cited by Homen, E. A., and Laitinen, T.: Die Wirkung von Streptokokken und ihrer Toxine auf periphere Nerven, Spinal ganglien und das Rückenmark. Beitr. z. path. Anat. und allg. Path., 1899, 25. 4-96. 
34. Nuzum, J. W.: Bacteriologic findings in cerebrospinal fluid in poliomyelitis. Jour. Am. Med. Assn., 1916, 67, 1437-1439.

35. Nuzum, J. W., and Herzog, M.: Experimental studies in the etiology of acute epidemic poliomyelitis. Jour. Am. Med. Assn., 1916, 67, 1205-1208.

36. Pasteur, W., Foulerton, A. G. R., and MacCormac, H.: On a case of acute poliomyelitis associated with a diplococcal infection of the spinal sac. Lancet, $1908,1,484-487$.

37. Potpeschnigg, K.: Bakteriologische Untersuchungsergebnisse bei Poliomyelitis (Heine-Medin'sche Krankheit). Wien klin. Wchnschr., 1909, 22, 1334. Cited by Römer, P. H., loc. cit.

38. Poynton, F. J., and Paine, A.: The etiology of rheumatic fever. Lancet, $1900,2,861-869 ; 932-935$.

39. Robertson, H. E., and Chesley, A. J.: Pathology and bacteriology of acute anterior poliomyelitis. Arch. Int. Med., 1910, 6, 233-269.

40. Roger, H.: Atrophie musculaire progressive experimentale. Ann. de l'Inst. Pasteur, 1892, 6, 436-450.

41. Römer, P. H.: Epidemic infantile paralysis. Eng. Trans. New York, Wood, 1913, 208 pp.

42. Römer. P. H.: Untersuchungen zur Aetiologie der epidemischen Kinderlähmung. München. med. Wchnschr., 1909, 56, 2505-2508.

43. Rosenau, M. J., and Havens, L. C.: Experiments with poliomyelitis in the rabbit. Jour. Exper. Med., 1916, 23, 461-474.

44. Rosenow, E. C.: The newer bacteriology of various infections as determined by special methods. Jour. Am. Med. Assn., 1914, 53, 903-908.

45. Rosenow, E. C.: Elective localization of streptococci. Jour. Am. Med. Assn., 1915, 65, 1687-91.

46. Rosenow, E. C.: Elective localization of bacteria in diseases of the nervous system. Jour. Am. Med. Assn., 1916, 67, 662-666.

47. Rosenow, E. C., and Gray, H.: Agglutination of the pleomorphic streptococcus isolated from epidemic poliomyelitis by immune horse, human, and monkey serum. Jour. Infect. Dis., 1918, 22, 345-376.

48. Rosenow, E. C., Towne, E. B., and Wheeler, G. W.: The etiology of epidemic poliomyelitis. Preliminary note. Jour. Am. Med. Assn., 1916, 67, $1202-1205$.

49. Rosenow, E. C., Towne, E. B., and Wheeler, G. W.: Observations on immunity of monkeys to experimental poliomyelitis. Jour. Am. Med. Assn., 1917, 68, 280-282.

50. Schultze, F.: Zur Aetiologie der acuten Poliomyelitis. München. med. Wchnschr., 1898, 45, 1197-1199.

51. Wickman, I.: Studien über Poliomyelitis acuta; zugleich ein Beitrag zur Kenntnis der myelitis acuta. Arbeiten aus dem pathologie. Institut der Universitat Helsingfors (Finland), 1905, 1, 109-293.

52. Wickman, I.: Acute poliomyelitis. Eng. trans. New York, Jour. Nervous and Mental Dis. Pub. Co., 1913, 135 pp.

53. Vidal and Besanzon: Ann. de 1'Inst. Pasteur, 1895, p. 104 . Cited by Homen, E. A., and Laitinen, T.: Die Wirkung von Streptokokken und ihrer Toxine auf periphere Nerven, Spinalganglien und das Rückenmark. Beitr. z. path. Anat. u. z. allg. Path., 1899, 25, 4-96. 


\section{EXPLANATION OF PLATES 9-13}

Fig. 1.-Guinea-pig 262 and normal control. Flaccid paralysis of hind legs and muscles of the back 3 days after the intravenous injection of the primary culture from the tonsil (see protocol).

Fig. 2,-Guinea-pig 264. Flaccid paralysis of the left foreleg 24 hours after the intravenous injection of pleomorphic streptococcus from a single colony on blood agar from tonsil pus (see protocol).

Fig. 3.-Guinea-pig 337. Paralysis of the left hind leg with contractures and wasting of the hind quarters 14 days after intravenous injection of the pleomorphic streptococcus from a single colony from the cord of Case 70\%, after one animal passage (see protocol).

Fig. 4.-Guinea-pig 384. Flaccid paralysis of the hind legs and right foreleg with marked weakness of the muscles of the back, 3 days after intravenous injection of the streptococcus from the tonsil after one animal passage (see protocol).

Fig. 5.--Guinea-pig 396. Flaccid paralysis of the right hind leg 48 hours after intravenous injection of streptococcus from the brain of Case 714, after two animal passages (see protocol and text Figure 1).

Fig. 6.-Marked perivascular round-cell infiltration of blood vessels in the brain of Guinea-pig 374. Hematoxylin and eosin. $\times 200$.

Fig. 7.-Streptococci in the area of perivascular infiltration shown in Figure 6. GramWeigert. $\times 1000$.

Fig. 8.-Streptococci adjacent to the area of hemorrhage in the cord of Guinea-pig 352 . Gram-Weigert. $\times 1000$.

Fig. 9.-Diplococci adjacent to the dilated capillary in the cord of the guinea-pig injected intravenously 5 days previously with a culture from the spinal fluid of Guinea-pig 324 . Gram-Weigert. $\times 1000$.

Fig. 10.-Rabbit 952. Flaccid paralysis of Aug. 6, 1916, 4 days after intravenous injection of the primary culture from the tonsil of Case 693 .

Fig. 11.-Rabbit 999. Flaccid paralysis of the extremities, 4 days after intravenous injection (see protocol).

Fig. 12.--Rabbit 1017. Flaccid paralysis of fore extremities, 2 days after intravenous injection (see protocol).

Fig. 13.-Hemorrhage and leukocytic infiltration of the pia and round-cell infiltration in the anterior horn of the spinal cord in rabbit shown in Figure 12. Methylene blue and eosin. $\times 50$.

Fig. 14.-Rabbit 939. Round-cell infiltration of the cerebellum 16 days after intravenous injection of the streptococcus from the tonsil of Case 686. Methylene blue and cosin. $\times 100$.

Fig. 15.-Diffuse and perivascular round-cell infiltration in the cerebellum of Rabbit 1001 (see protocol). Methylene blue and eosin. $\times 50$.

Fig. 16.-Same as Figure 15. Higher magnification of blood vessel showing round-cell infiltration. Methylene blue and eosin. $\times 1000$.

Fig. 17.-Round-cell infiltration in the cerebellum of Rabbit 985 (see protocol). Hema. toxylin and eosin. $\times 250$.

Fig. 18. - Chain of streptococci in the anterior horn of the spinal cord of Rabbit.980 (see protocol). Gram-Weigert. $\times 1000$.

Fig. 19.-Circumscribed and perivascular infiltration by round cells in the pons of Rabbit 1018 (see protocol). Methylene blue and eosin. $\times 200$.

Fig. 20.-Diplococcus adjacent to the area of hemorrhage in the brain of a rabbit 3 days after intracerebral inoculation with Berkefeld filtrate of the brain of Monkey 10 (see text Figure 1). Gram-Weigert. $\times 1000$. 


\section{Plate 9}
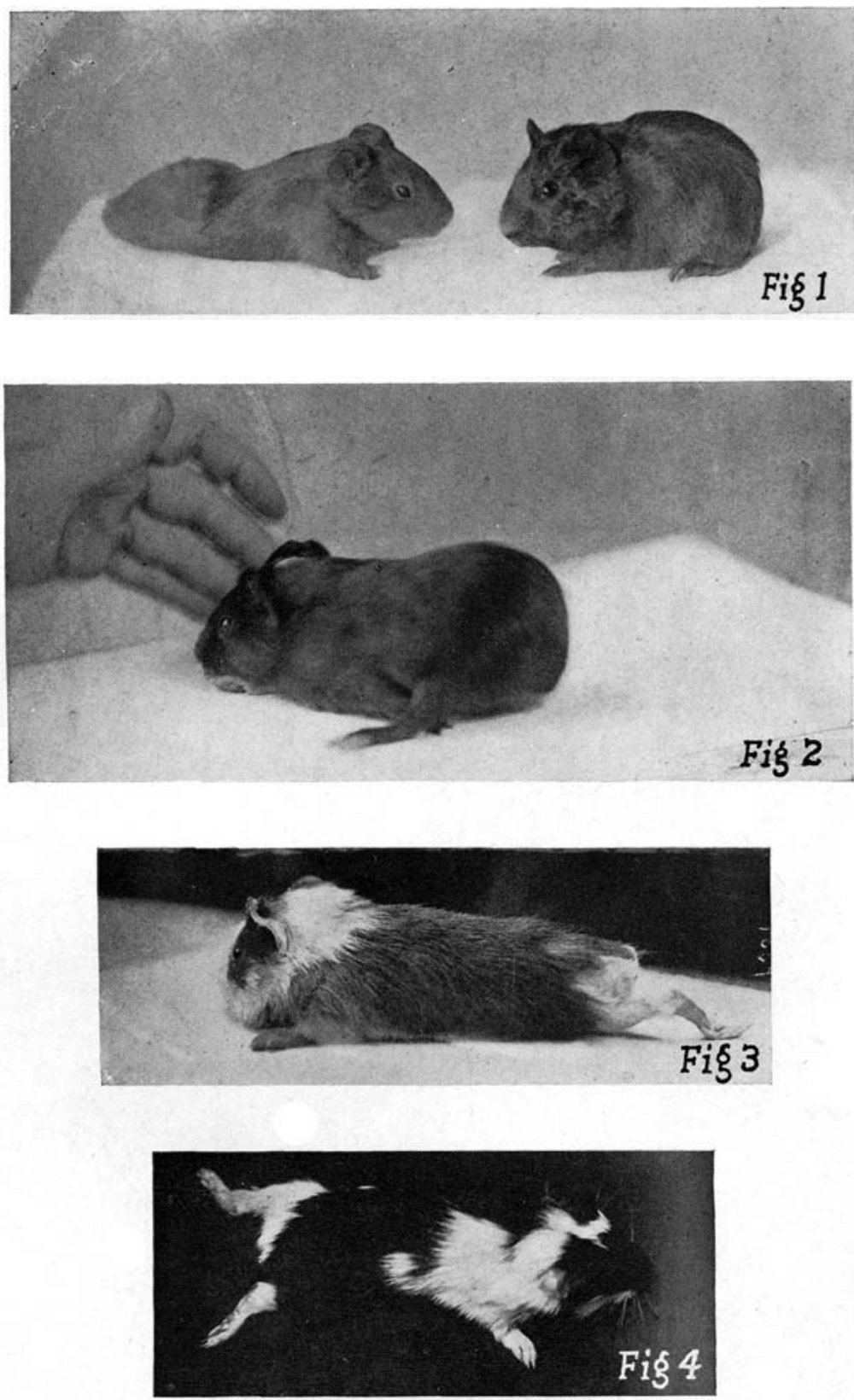

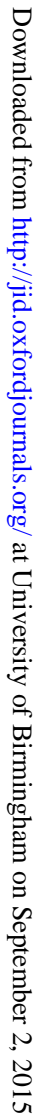

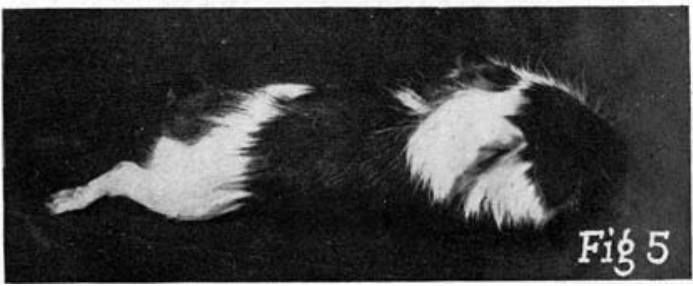


Plate 10
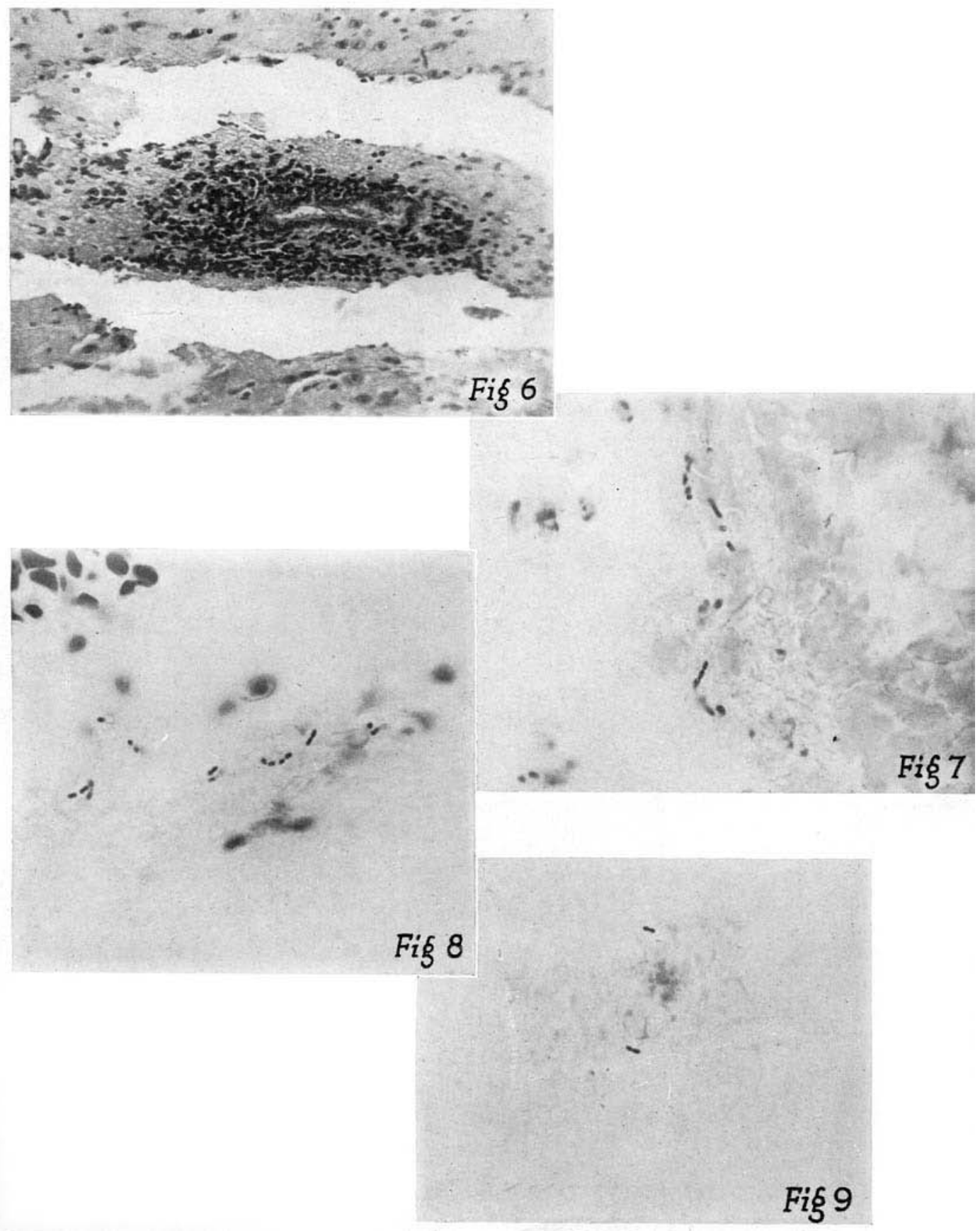


\section{Plate 11}
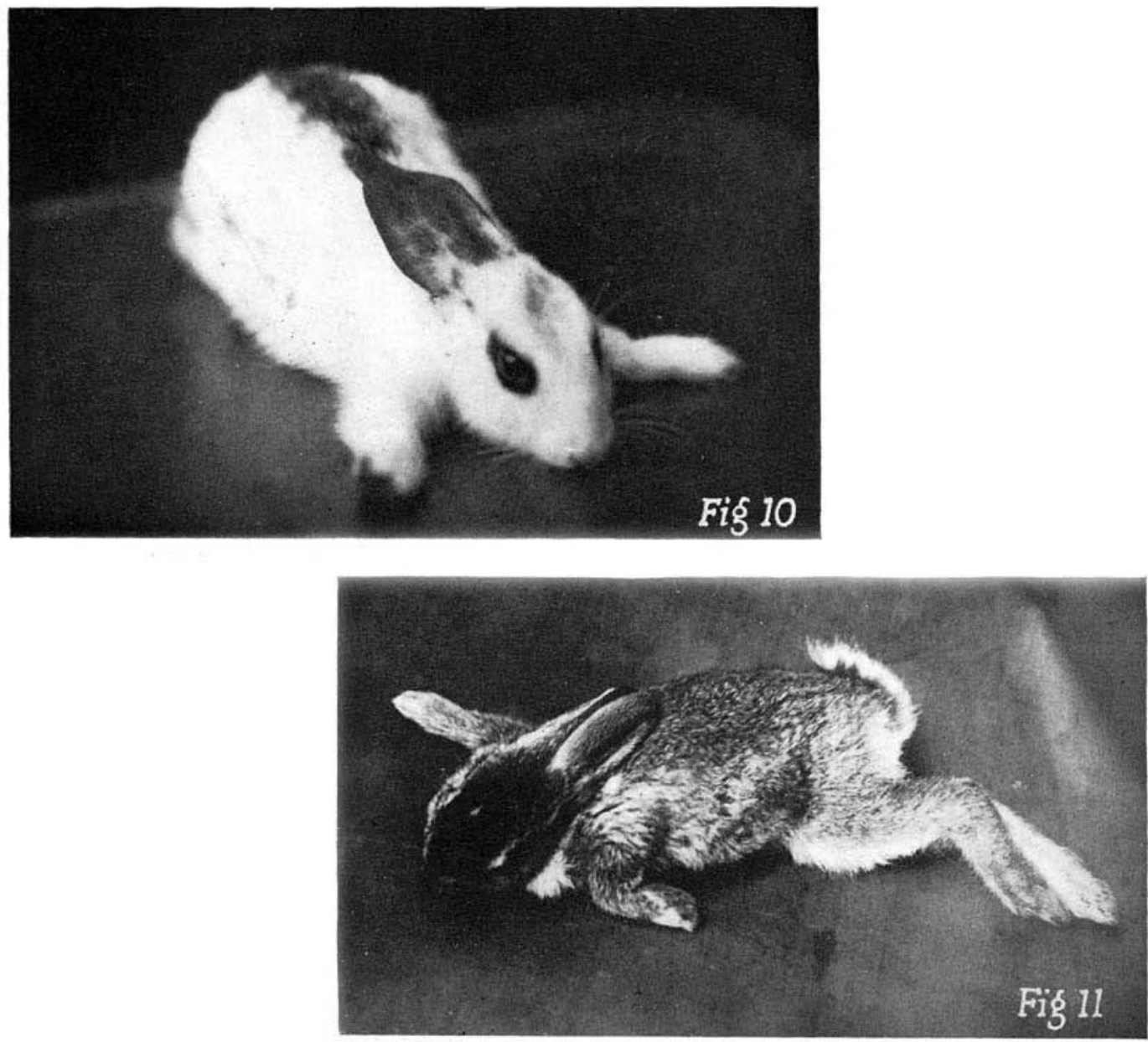

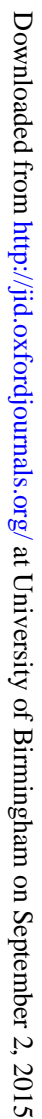

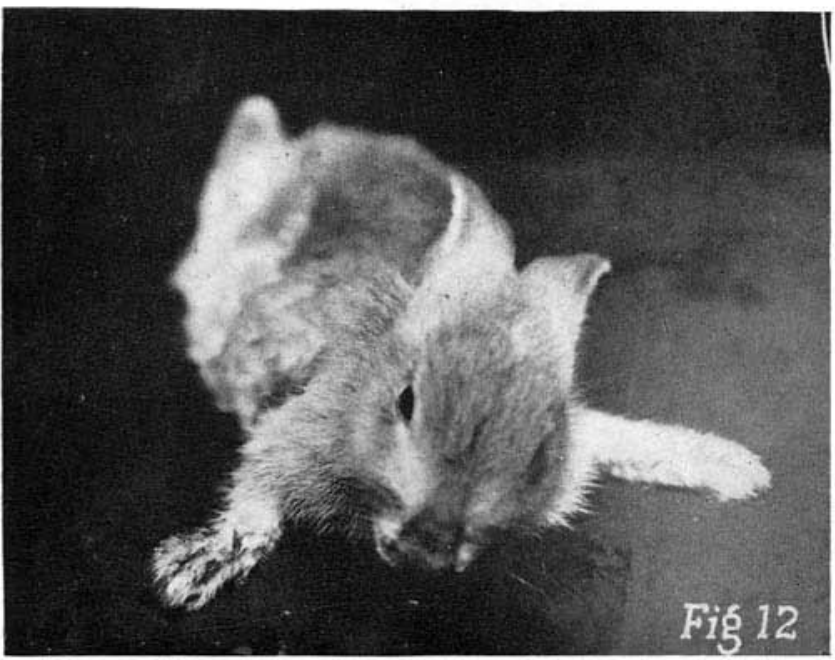


Plate 12

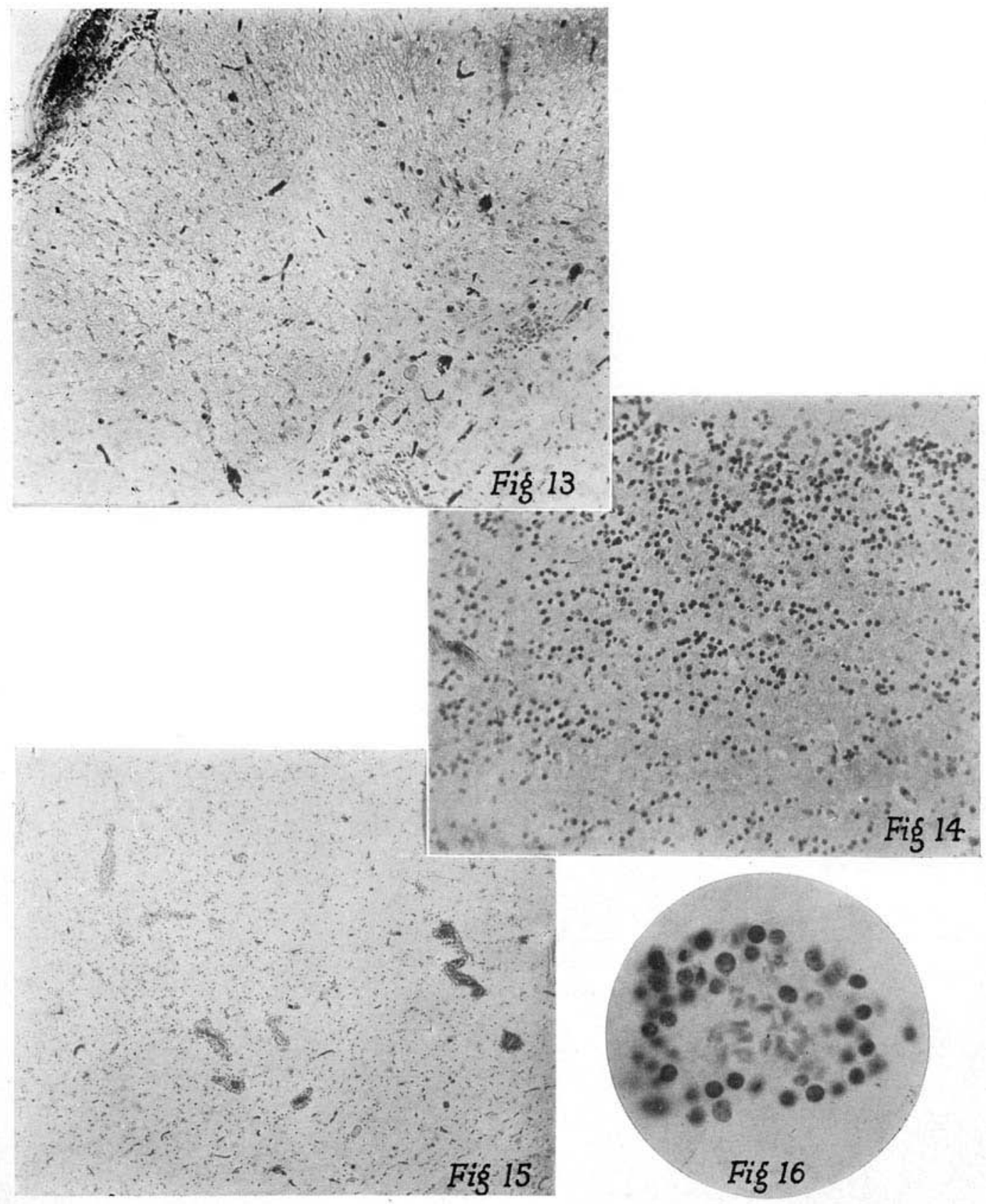

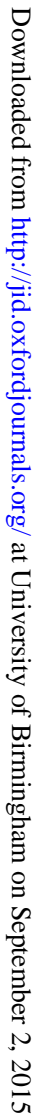


Plate 13
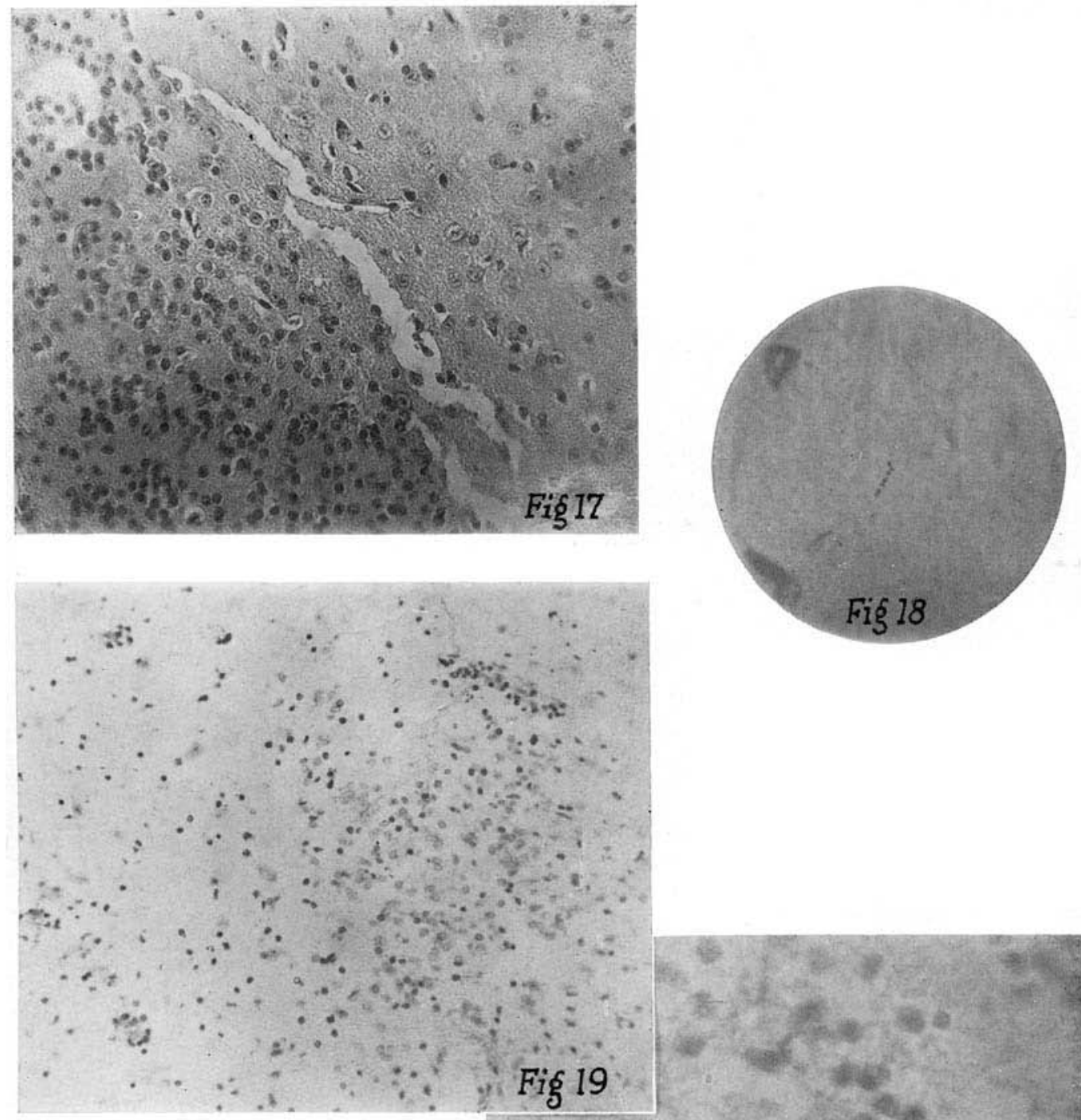

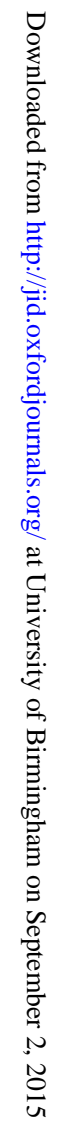

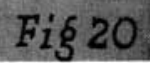

